\title{
ASYMPTOTICS FOR A SYSTEM OF NONLINEARLY COUPLED WAVE EQUATIONS WITH AN APPLICATION TO THE GALLOPING OSCILLATIONS OF OVERHEAD TRANSMISSION LINES*
}

By

\author{
W. T. VAN HORSSEN
}

Delft University of Technology, The Netherlands

\begin{abstract}
In this paper an asymptotic theory for a class of initial-boundary value problems for systems of weakly and nonlinearly coupled wave equations is presented. The theory implies the well-posedness of the problem in the classical sense and the asymptotic validity of formal approximations on long time scales.

As an application of the theory, an initial-boundary value problem for a system of weakly and nonlinearly coupled wave equations is studied in detail using a twotime-scales perturbation method. From an aero-elastic analysis it is shown that this initial-boundary value problem may be regarded as a model describing the galloping oscillations of overhead transmission lines in the vertical and in the horizontal direction.
\end{abstract}

1. Introduction. In this paper an asymptotic theory is presented for the following initial-boundary value problem for a system of nonlinearly perturbed wave equations

$$
\begin{gathered}
\mathbf{u}_{t}-C \mathbf{u}_{x x}+\varepsilon \mathbf{f}\left(x, t, \mathbf{u}, \mathbf{u}_{t}, \mathbf{u}_{x} ; \varepsilon\right)=\mathbf{0}, \quad 0<x<\pi, t>0, \\
\mathbf{u}(x, 0 ; \varepsilon)=\mathbf{u}_{0}(x ; \varepsilon) \quad \text { and } \quad \mathbf{u}_{t}(x, 0 ; \varepsilon)=\mathbf{u}_{1}(x ; \varepsilon), \quad 0<x<\pi, \\
\mathbf{u}(0, t ; \varepsilon)=\mathbf{u}(\pi, t ; \varepsilon)=\mathbf{0}, \quad t \geq 0,
\end{gathered}
$$

with $\mathbf{u}=\left(u_{1}, u_{2}, \ldots, u_{n}\right)^{\mathrm{T}}, \mathbf{f}=\left(f_{1}, f_{2}, \ldots, f_{n}\right)^{\mathrm{T}}$ and $0<|\varepsilon| \leq \varepsilon_{0} \ll 1$. The $(n \times n)$ diagonal matrix $C$ has $\varepsilon$-independent diagonal elements $c_{i i}^{2}(i=1,2, \ldots, n)$ with $c_{i i}>0$, and the functions $\mathbf{f}, \mathbf{u}_{0}$, and $\mathbf{u}_{1}$ have to satisfy certain smoothness properties, which are mentioned in Sec. 2. As usual the derivative of a matrix-valued function is obtained by taking the derivative of each element of the matrix-valued function. The asymptotic theory presented here implies the well-posedness in the classical sense of the initial-boundary value problem (1.1)-(1.3) as well as the asymptotic validity of formal approximations. In this paper formal approximations are defined to be vectorvalued functions satisfying the differential equations and the initial conditions up to some order depending on the small parameter $\varepsilon$.

For scalar-valued functions similar asymptotic theories have been developed in [6] for an initial-boundary value problem for the weakly semi-linear telegraph equation

*Received January 22, 1988.

(C)1989 Brown University 
$u_{t t}-u_{x x}+u+\varepsilon f(x, t, u ; \varepsilon)=0$, and in [7] for an initial-boundary value problem for the weakly nonlinear wave equation $u_{t t}-u_{x x}+\varepsilon f\left(x, t, u, u_{t}, u_{x} ; \varepsilon\right)=0$. Both types of equations were considered subject to the initial values $u(x, 0 ; \varepsilon)=u_{0}(x ; \varepsilon)$ and $u_{t}(x, 0 ; \varepsilon)=u_{1}(x ; \varepsilon)$ and the boundary values $u(0, t ; \varepsilon)=u(\pi, t ; \varepsilon)=0$. The well-posedness in the classical sense and the asymptotic validity of a class of formal approximations could be obtained on a time scale of order $|\varepsilon|^{-1 / 2}$ for the problem for the weakly semi-linear telegraph equation and could be established on a time scale of order $|\varepsilon|^{-1}$ for the problem for the weakly nonlinear wave equation. For the initial-boundary value problem (1.1)-(1.3) it will be shown that a time scale of order $|\varepsilon|^{-1}$ can be obtained.

The asymptotic theory in $[6,7]$ and the asymptotic theory presented in this paper can be regarded as an extension of the asymptotic theory for ordinary differential equations as for instance described in $[1,2,4,14]$. Moreover, the asymptotic results presented in this paper can be seen as a generalization of the asymptotic results obtained in [7].

This paper, being an attempt to contribute to the foundations of the asymptotic methods for (systems of) weakly nonlinear hyperbolic partial differential equations, is organized as follows. In Sec. 2 the well-posedness of the problem is investigated and established on a time scale of order $|\varepsilon|^{-1}$ and in Sec. 3 the asymptotic validity of formal approximations is studied, that is, estimates of the differences between the solution and the formal approximations are given on a time scale for which the problem has been shown to be well-posed. The asymptotic theory is applied in Sec. 5 to the initial-boundary value problem $(1.1)-(1.3)$ with $\mathbf{u}=(v, w)^{\mathrm{T}}$ and

$$
\mathbf{f}\left(x, t, \mathbf{u}, \mathbf{u}_{t}, \mathbf{u}_{x} ; \varepsilon\right) \equiv\left(\begin{array}{l}
a_{10} v_{t}+a_{01} w_{t}+a_{20} v_{t}^{2}+a_{11} v_{t} w_{t}+a_{02} w_{t}^{2}+a_{03} w_{t}^{3} \\
b_{01} w_{t}+b_{11} v_{t} w_{t}+b_{02} w_{t}^{2}+b_{03} w_{t}^{3}
\end{array}\right),
$$

where $a_{10}, a_{01}, \ldots, b_{03}$ are constants independent of $\varepsilon$. In Sec. 4 it follows from an aero-elastic analysis that this initial-boundary value problem may be regarded as a model which describes the growth of wind-induced oscillations of overhead transmission lines in the vertical and in the horizontal direction. In fact this initial-boundary value problem is an extension of a model (describing only the vertical displacements of the transmission lines) which has been (postulated in the early seventies in [11, 12] and which recently has been derived in [7]. From a practical point of view it is interesting to investigate the vertical as well as the horizontal displacements of the transmission line, since one or both of the displacements may give rise to conductor damage due to impact of conductor lines or due to flashover as a result of a phase difference between conductor lines, for which the mutual distance has become too small.

Using a two-time-scale perturbation method, as for instance successfully used in $[3,6,7,9,10]$, an asymptotic approximation of the solution of the aforementioned initial-boundary value problem will be constructed. In some sense it is remarkable that the two-time-scale perturbation method as developed in $[3,10]$ and applied in $[3,6,7,9,10]$ to an initial-boundary value problem for a single perturbed wave equation may also be used for an initial-boundary value problem for the aforementioned 
system of perturbed wave equations. Finally, in Sec. 6 some of the results obtained in this paper will be discussed.

2. The well-posedness of the problem. In this section a weakly nonlinear initialboundary value problem for a vector-valued function $\mathbf{u}(x, t ; \varepsilon)$ will be considered. As usual a derivative of a vector-valued function is obtained by taking the derivative of each element of the vector-valued function. Furthermore, a vector-valued function is said to be continuous (or differentiable) if and only if all the elements of the vector-valued function are continuous (or differentiable). Let $\mathbf{u}(x, t ; \varepsilon) \equiv$ $\left(u_{1}(x, t ; \varepsilon), u_{2}(x, t ; \varepsilon), \ldots, u_{n}(x, t ; \varepsilon)\right)^{\mathrm{T}}$ with $1 \leq n<\infty$ and let $\mathbf{u}, \mathbf{u}_{t}, \mathbf{u}_{x}, \mathbf{u}_{t t}, \mathbf{u}_{t x}=\mathbf{u}_{x t}$ and $\mathbf{u}_{x x}$ be continuous on $0 \leq x \leq \pi$ and $t \geq 0$. The following weakly nonlinear initial-boundary value problem for the vector-valued function $\mathbf{u}(x, t ; \varepsilon)$ will now be considered:

$$
\begin{array}{ll}
\mathbf{u}_{t t}-C \mathbf{u}_{x x}+\varepsilon \mathbf{F}(\mathbf{u} ; \varepsilon)=\mathbf{0}, & 0<x<\pi, t>0, \\
\mathbf{u}(x, 0 ; \varepsilon)=\mathbf{u}_{0}(x ; \varepsilon), & 0<x<\pi, \\
\mathbf{u}_{t}(x, 0 ; \varepsilon)=\mathbf{u}_{1}(x ; \varepsilon), & 0<x<\pi, \\
\mathbf{u}(0, t ; \varepsilon)=\mathbf{u}(\pi, t ; \varepsilon)=\mathbf{0}, & t \geq 0,
\end{array}
$$

where $C$ is an $(n \times n)$ diagonal matrix with $\varepsilon$-independent diagonal elements $c_{i i}^{2}$ $(i=1,2, \ldots, n)$ and $c_{i i}>0$,

$$
\mathbf{F}(\mathbf{u} ; \varepsilon)(x, t) \equiv \mathbf{f}\left(x, t, \mathbf{u}(x, t ; \varepsilon), \mathbf{u}_{t}(x, t ; \varepsilon), \mathbf{u}_{x}(x, t ; \varepsilon) ; \varepsilon\right),
$$

$0<|\varepsilon| \leq \varepsilon_{0} \ll 1$, and where $\mathbf{u}_{0}(x ; \varepsilon)=\left(u_{01}(x ; \varepsilon), \ldots, u_{0 n}(x ; \varepsilon)\right)^{\mathrm{T}}, \mathbf{u}_{1}(x ; \varepsilon)=\left(u_{11}(x ; \varepsilon)\right.$, $\left.\ldots, u_{1 n}(x ; \varepsilon)\right)^{\mathrm{T}}$ and $\mathbf{f}(x, t, \mathbf{u}, \mathbf{p}, \mathbf{q} ; \varepsilon)=\left(f_{1}(x, t, \mathbf{u}, \mathbf{p}, \mathbf{q} ; \varepsilon), \ldots, f_{n}(x, t, \mathbf{u}, \mathbf{p}, \mathbf{q} ; \varepsilon)\right)^{\mathrm{T}}$ with $\mathbf{u}=\left(u_{1}, \ldots, u_{n}\right)^{\mathrm{T}}, \mathbf{p}=\left(p_{1}, \ldots, p_{n}\right)^{\mathrm{T}}$ and $\mathbf{q}=\left(q_{1}, \ldots, q_{n}\right)^{\mathrm{T}}$ satisfying

$$
u_{0 i}, \frac{\partial u_{0 i}}{\partial x}, \frac{\partial^{2} u_{0 i}}{\partial x^{2}} \in C\left([0, \pi] \times\left[-\varepsilon_{0}, \varepsilon_{0}\right], \mathbb{R}\right) \text { for } i=1,2, \ldots, n \text {, }
$$

with

$$
\mathbf{u}_{0}(0 ; \varepsilon)=\mathbf{u}_{0}(\pi ; \varepsilon)=\frac{\partial^{2} \mathbf{u}_{0}(0 ; \varepsilon)}{\partial x^{2}}=\frac{\partial^{2} \mathbf{u}_{0}(\pi ; \varepsilon)}{\partial x^{2}}=\mathbf{0}
$$

$u_{1 i}, \frac{\partial u_{1 i}}{\partial x} \in C\left([0, \pi] \times\left[-\varepsilon_{0}, \varepsilon_{0}\right], \mathbb{R}\right)$ for $i=1,2, \ldots, n$, with $\mathbf{u}_{1}(0 ; \varepsilon)=\mathbf{u}_{1}(\pi ; \varepsilon)=\mathbf{0}$, and

$f_{i}, \frac{\partial f_{i}}{\partial x}, \frac{\partial f_{i}}{\partial u_{j}}, \frac{\partial f_{i}}{\partial p_{j}}, \frac{\partial f_{i}}{\partial q_{j}} \in C\left([0, \pi] \times[0, \infty\rangle \times \mathbb{R}^{3 n} \times\left[-\varepsilon_{0}, \varepsilon_{0}\right], \mathbb{R}\right) \quad$ for $i, j=1,2, \ldots, n$,

with

$$
\mathbf{F}(\mathbf{u} ; \varepsilon)(0, t)=\mathbf{F}(\mathbf{u} ; \varepsilon)(\pi, t)=\mathbf{0} \text { for } t \geq 0 .
$$

Furthermore, $\mathbf{f}(x, t, u, p, q ; \varepsilon)$ and its partial derivatives with respect to $x, u, p$, and $q$ are assumed to be uniformly bounded for those values of $t$ under consideration. To prove in the classical sense existence and uniqueness of the solution of the initialboundary value problem (2.1)-(2.4) an equivalent system of coupled integral equations will be used. In order to derive this system of integral equations the initialboundary value problem is transformed into an initial value problem by extending 
the vector-valued functions $\mathbf{u}, \mathbf{f}, \mathbf{u}_{0}$, and $\mathbf{u}_{1}$ in $x$ to odd and $2 \pi$-periodic functions (see for instance [16], Chap. 2). The extensions of $\mathbf{u}, \mathbf{f}, \mathbf{u}_{0}$, and $\mathbf{u}_{1}$ are denoted by $\mathbf{u}^{*}, \mathbf{f}^{*}, \mathbf{u}_{0}^{*}$, and $\mathbf{u}_{1}^{*}$, respectively. Then, assuming that $\mathbf{u}^{*}, \mathbf{u}_{t}^{*}, \mathbf{u}_{x}^{*}, \mathbf{u}_{t t}^{*}, \mathbf{u}_{t x}^{*}=\mathbf{u}_{x t}^{*}$ and $\mathbf{u}_{x x}^{*}$ are continuous on $-\infty<x<\infty$ and $t \geq 0$, an integral representation for the solution $\mathbf{u}^{*}$ of the initial value problem is given by

$$
\mathbf{u}^{*}(x, t ; \varepsilon)=-\frac{\varepsilon}{2} \int_{0}^{t} \mathbf{I f}^{*}(\tau ; x, t) d \tau+\mathbf{u}_{l}^{*}(x, t ; \varepsilon),
$$

where $\mathbf{I f}^{*}(\tau ; x, t)$ is a vector with elements $I f_{i}^{*}(\tau ; x, t)$ defined by

$$
I f_{i}^{*}(\tau ; x, t)=\frac{1}{c_{i i}} \int_{x-c_{11}(t-\tau)}^{x+c_{u}(t-\tau)} f_{i}^{*}\left(\xi, \tau, \mathbf{u}^{*}(\xi, \tau ; \varepsilon), \mathbf{u}_{t}^{*}(\xi, \tau ; \varepsilon), \mathbf{u}_{\xi}^{*}(\xi, \tau ; \varepsilon) ; \varepsilon\right) d \xi
$$

for $i=1,2, \ldots, n$, and where $\mathbf{u}_{l}^{*}(x, t ; \varepsilon)$ is a vector with elements $u_{l i}^{*}(x, t ; \varepsilon)$ defined by

$$
u_{l i}^{*}(x, t ; \varepsilon)=\frac{1}{2} u_{0 i}^{*}\left(x+c_{i i} t ; \varepsilon\right)+\frac{1}{2} u_{0 i}^{*}\left(x-c_{i i} t ; \varepsilon\right)+\frac{1}{2 c_{i i}} \int_{x-c_{i i} t}^{x+c_{i i} t} u_{1 i}^{*}(\xi ; \varepsilon) d \xi
$$

for $i=1,2, \ldots, n$. As usual the integral of a matrix-valued function is obtained by taking the integral of each element of the matrix-valued function. Using reflection principles, (2.9) can be rewritten as a system of coupled integral equations on the semi-infinite strip $0 \leq x \leq \pi, 0 \leq t<\infty$, yielding

$$
\mathbf{u}(x, t ; \varepsilon)=\frac{\varepsilon}{2} \int_{0}^{t} \int_{0}^{\pi} G(\xi, \tau ; x, t) \mathbf{F}(\mathbf{u} ; \varepsilon)(\xi, \tau) d \xi d \tau+\mathbf{u}_{l}(x, t ; \varepsilon),
$$

where $G(\xi, \tau ; x, t)$ is the $(n \times n)$ diagonal matrix with diagonal elements

$$
\begin{array}{r}
g_{i i}(\xi, \tau ; x, t)=\frac{1}{c_{i i}} \sum_{k \in \mathbb{Z}}\left\{H\left(c_{i i}(t-\tau)-\xi+2 k \pi-x\right) H\left(c_{i i}(t-\tau)+\xi-2 k \pi+x\right)\right. \\
\left.-H\left(c_{i i}(t-\tau)+\xi+2 k \pi-x\right) H\left(c_{i i}(t-\tau)-\xi-2 k \pi+x\right)\right\}
\end{array}
$$

for $i=1,2, \ldots, n$, and where $\mathbf{u}_{l}$ is given by

$$
\mathbf{u}_{l}(x, t ; \varepsilon)=\frac{1}{2} \int_{0}^{\pi}\left\{\frac{\partial G}{\partial \tau}(\xi, 0 ; x, t) \mathbf{u}_{0}(\xi ; \varepsilon)-G(\xi, 0 ; x, t) \mathbf{u}_{1}(\xi ; \varepsilon)\right\} d \xi .
$$

The function $H(a)$ on $\mathbb{R}$ is equal to 1 for $a>0, \frac{1}{2}$ for $a=0$, and zero otherwise. In (2.12) it is assumed that $g_{i i}$ is differentiated according to the rule

$(d / d \tau)\{H(f(\tau)) H(g(\tau))\}=\delta_{0}(f(\tau))(d f(\tau) / d \tau) H(g(\tau))+H(f(\tau)) \delta_{0}(g(\tau))(d g(\tau) / d \tau)$

where $\delta_{0}$ is the Dirac delta function. In fact, $g_{i i}$ as defined by (2.11) is the Green's function for the differential operator $\partial^{2} / \partial t^{2}-c_{i i}^{2} \partial^{2} / \partial x^{2}$ and the Dirichlet boundary conditions. And so, $G$ can be identified with the matrix-valued function of Green for the differential operator $\partial^{2} / \partial t^{2}-C \partial^{2} / \partial x^{2}$ and the boundary conditions (2.4). It is also worth noticing that the solution of the (linear) initial-boundary value problem (2.1) $-(2.4)$ with $\mathbf{F} \equiv \mathbf{0}$ is given by $\mathbf{u}_{l}(x, t ; \varepsilon)$.

Elementary calculations show that if $\mathbf{v}(x, t ; \varepsilon)$ is a twice continuously differentiable solution of the initial-boundary value problem $(2.1)-(2.4)$ then $\mathbf{v}(x, t ; \varepsilon)$ is a solution 
of the system of integral equations (2.10). Also, if $\mathbf{w}(x, t ; \varepsilon)$ is a twice continuously differentiable solution of the system of integral equations (2.10) then it can easily be shown that $\mathbf{w}(x, t ; \varepsilon)$ is a solution of the initial-boundary value problem (2.1)(2.4). Hence, the system of integral equations (2.10) and the initial-boundary value problem (2.1)-(2.4) are equivalent if twice continuously differentiable solutions exist, that is, if vector-valued functions exist of which all elements are twice continuously differentiable. Now it will be proved that a unique, twice continuously differentiable solution of the system of integral equations (2.10) exists in a strip $\Omega_{L}$ of the $(x, t)$ plane. So, a unique and twice continuously differentiable solution exists for the initial-boundary value problem (2.1)-(2.4) on $\Omega_{L}$.

In order to prove existence and uniqueness in the classical sense of the solution of the system of nonlinear integral equations $(2.10)$ a fixed point theorem will be used. Let $\Omega_{L}$ be given by

$$
\Omega_{L}=[0, \pi] \times\left[0, L|\varepsilon|^{-1}\right]
$$

in which $L$ is a sufficiently small, positive constant independent of $\varepsilon$. Let $C_{M}^{2}\left(\Omega_{L}, \mathbb{R}^{n}\right)$ be the space of all real-valued and twice continuously differentiable functions $\mathbf{w}(x, t)$ $=\left(w_{1}(x, t), w_{2}(x, t), \ldots, w_{n}(x, t)\right)^{\mathrm{T}}$ on $\Omega_{L}$ with norm $\|\cdot\|_{C_{M}^{2}}$ defined by:

$$
\|\mathbf{w}\|_{C_{M}^{2}}=\sum_{i=1}^{n} \sum_{\substack{k, l=0 \\ k+l \leq 2}}^{2} \max _{(x, t) \in \Omega_{L}}\left|\frac{\partial^{k+l} w_{i}(x, t)}{\partial x^{k} \partial t^{l}}\right| \leq M .
$$

From the smoothness properties of $\mathbf{u}_{0}$ and $\mathbf{u}_{1}$ it follows that (for fixed $\mathbf{u}_{0}$ and $\mathbf{u}_{1}$ ) there exists a positive constant $M_{1}$ independent of $\varepsilon$ such that

$$
\left\|\mathbf{u}_{l}\right\|_{C_{M_{1}}^{2}} \leq \frac{1}{2} M_{1}
$$

and from the smoothness properties of $\mathbf{F}$ (as given by (2.5) and (2.8)) it follows that there exist $\varepsilon$-independent constants $M_{2}$ and $M_{3}$ such that

$$
\begin{gathered}
\sum_{k=0}^{1}\left|\frac{d^{k}}{d x^{k}} f_{i}\left(x, t, \mathbf{v}(x, t), \mathbf{v}_{t}(x, t), \mathbf{v}_{x}(x, t) ; \varepsilon\right)\right| \leq M_{2} \\
\sum_{k=0}^{1}\left|\frac{d^{k}}{d x^{k}}\left(f_{i}\left(x, t, \mathbf{v}(x, t), \mathbf{v}_{t}(x, t), \mathbf{v}_{x}(x, t) ; \varepsilon\right)-f_{i}\left(x, t, \mathbf{w}(x, t), \mathbf{w}_{t}(x, t), \mathbf{w}_{x}(x, t) ; \varepsilon\right)\right)\right| \\
\leq M_{3}\|\mathbf{v}-\mathbf{w}\|_{C_{M_{1}}^{2},}
\end{gathered}
$$

for all $(x, t) \in \Omega_{L}$ and $i=1,2, \ldots, n, \varepsilon \in\left[-\varepsilon_{0}, \varepsilon_{0}\right]$ and $\mathbf{v}, \mathbf{w} \in C_{M_{1}}^{2}\left(\Omega_{L}, \mathbb{R}^{n}\right)$. Now let the integral operator $T: C^{2}\left(\Omega_{L}, \mathbb{R}^{n}\right) \rightarrow C^{2}\left(\Omega_{L}, \mathbb{R}^{n}\right)$, which is related to the system of integral equations $(2.10)$, be defined by

$$
(T \mathbf{w})(x, t) \equiv \frac{\varepsilon}{2} \int_{0}^{t} \int_{0}^{\pi} G(\xi, \tau ; x, t) \mathbf{F}(\mathbf{w} ; \varepsilon)(\xi, \tau) d \xi d \tau+\mathbf{u}_{/}(x, t ; \varepsilon),
$$

where $G, \mathbf{F}$, and $\mathbf{u}_{\text {/ }}$ are given by (2.11), (2.5), and (2.12), respectively. According to Banach's fixed point theorem the integral operator $T$ has a unique fixed point in $C_{M_{1}}^{2}\left(\Omega_{L}, \mathbb{R}^{n}\right)$ if the operator $T$ maps $C_{M_{1}}^{2}\left(\Omega_{L}, \mathbb{R}^{n}\right)$ into itself and if $T$ is a contraction 
on $C_{M_{1}}^{2}\left(\Omega_{L}, \mathbb{R}^{n}\right)$. Now, it will be proved that the integral operator $T$ satisfies these two conditions. It is not difficult to show that $T$ maps $C_{M_{1}}^{2}\left(\Omega_{L}, \mathbb{R}^{n}\right)$ into the space of (real- and vector-valued) twice continuously differentiable functions on $\Omega_{L}$. In order to prove that $T$ maps $C_{M_{1}}^{2}\left(\Omega_{L}, \mathbb{R}^{n}\right)$ into itself estimates of the elements $g_{i i}(\xi, \tau ; x, t)$ of the diagonal matrix $G$ should be obtained for $0 \leq \xi \leq \pi, 0 \leq \tau \leq t$, fixed $x$ and $t$, and $i=1,2, \ldots, n$. It can be shown (see also [7]) that $\left|g_{i i}(\xi, \tau ; x, t)\right| \leq 1 / c_{i i}$ for $0 \leq \xi<\pi, \tau \geq 0$, fixed $x$ and $t$, and $i=1,2, \ldots, n$. Now let $\left(T \mathbf{v}-\mathbf{u}_{l}\right)_{i}$ be the $i$ th element $(i=1,2, \ldots, n)$ of the $(n \times 1)$-matrix $T \mathbf{v}-\mathbf{u}_{l}$. Using (2.13)-(2.15) and (2.17) and putting $c=\min \left(1, c_{11}, c_{22}, \ldots, c_{n n}\right)$ the following estimate can be made:

$$
\begin{aligned}
\|T \mathbf{v}\|_{C_{M_{M_{1}}}^{2}} & \leq\left\|T \mathbf{v}-\mathbf{u}_{/}\right\|_{C_{\bar{M}_{I_{1}}}^{2}}+\left\|\mathbf{u}_{/}\right\|_{C_{\dot{M}_{1}}^{2}} \\
& =\sum_{i=1}^{n} \sum_{\substack{k, l=0 \\
k+l \leq 2}}^{2} \max _{(x, t) \in \Omega_{L}}\left|\frac{\partial^{k+l}}{\partial x^{k} \partial t}\left((T \mathbf{v})(x, t)-\mathbf{u}_{l}(x, t ; \varepsilon)\right)_{i}\right|+\left\|\mathbf{u}_{l}\right\|_{C_{M_{M_{1}}}^{2}} \\
& \leq \frac{n}{c}\left(\left(\frac{\pi}{2}+5\right) M_{2} L+\varepsilon_{0} M_{2}\right)+\frac{1}{2} M_{1}
\end{aligned}
$$

for all $\mathbf{v} \in C_{M_{1}}^{2}\left(\Omega_{L}, \mathbb{R}^{n}\right)$. Now $\varepsilon_{0}$ has been assumed to be sufficiently small and so, there exists an $\varepsilon$-independent constant $L$ such that $(n / c)\left((\pi / 2+5) M_{2} L+\varepsilon_{0} M_{2}\right) \leq$ $\frac{1}{2} M_{1}$. Hence, $\|T \mathbf{v}\|_{C_{M_{1}}^{2}} \leq M_{1}$ for all $\mathbf{v} \in C_{M_{1}}^{2}\left(\Omega_{L}, \mathbb{R}^{n}\right)$. So, $T$ maps $C_{M_{1}}^{2}$ into itself. Using (2.13), (2.16), and (2.17) it will be shown that $T$ is a contraction on $C_{M_{1}}^{2}\left(\Omega_{L}, \mathbb{R}^{n}\right)$. Let $\mathbf{v}, \mathbf{w} \in C_{M_{1}}^{2}\left(\Omega_{L}, \mathbb{R}^{n}\right)$; then the following estimate can be obtained:

$$
\|T \mathbf{v}-T \mathbf{w}\|_{C_{M_{1}}^{2}} \leq \frac{n}{c}\left(\left(\frac{\pi}{2}+5\right) M_{3} L+\varepsilon_{0} M_{3}\right)\|\mathbf{v}-\mathbf{w}\|_{C_{M_{1}}^{2}} .
$$

It is clear that there exists an $\varepsilon$-independent constant $L$ such that $(n / c)((\pi / 2+$ 5) $\left.M_{3} L+\varepsilon_{0} M_{3}\right) \leq k<1$. Since there always exists a constant $L$ independent of $\varepsilon$ such that $(n / c)\left((\pi / 2+5) M_{2} L+\varepsilon_{0} M_{2}\right) \leq \frac{1}{2} M_{1}$ and $(n / c)\left((\pi / 2+5) M_{3} L+\varepsilon_{0} M_{3}\right) \leq k<1$, it follows that $T$ maps $C_{M_{1}}^{2}\left(\Omega_{L}, \mathbb{R}^{n}\right)$ into itself and is a contraction. Banach's fixed point theorem then implies that $T$ has a unique fixed point in $C_{M_{1}}^{2}\left(\Omega_{L}, \mathbb{R}^{n}\right)$, that is, a unique and twice continuously differentiable function on $\Omega_{L}$. Hence, the solution of the system of the integral equations $(2.10)$ is unique and twice continuously differentiable on $\Omega_{L}$. So, on $\Omega_{L}$, a unique and twice continuously differentiable solution exists for the initial-boundary value problem (2.1)-(2.4).

Next it will be shown that the solution of the initial-boundary value problem (2.1)(2.4) depends continuously on the initial values. Let $\mathbf{u}(x, t ; \varepsilon)$ satisfy $(2.1)-(2.4)$ and let $\tilde{\mathbf{u}}(x, t ; \varepsilon)$ satisfy $(2.1),(2.4), \tilde{\mathbf{u}}(x, 0 ; \varepsilon)=\tilde{\mathbf{u}}_{0}(x ; \varepsilon)$ and $\tilde{\mathbf{u}}_{t}(x, 0 ; \varepsilon)=\tilde{\mathbf{u}}_{1}(x ; \varepsilon)$, where $\tilde{\mathbf{u}}_{0}$ and $\tilde{\mathbf{u}}_{1}$ satisfy (2.6) and (2.7). Let $\tilde{\mathbf{u}}_{/}$be given by

$$
\tilde{\mathbf{u}}_{l}(x, t ; \varepsilon)=\frac{1}{2} \int_{0}^{\pi}\left\{\frac{\partial G}{\partial \tau}(\xi, 0 ; x, \iota) \tilde{\mathbf{u}}_{0}(\xi ; \varepsilon)-G(\xi, 0 ; x, t) \tilde{\mathbf{u}}_{1}(\xi ; \varepsilon)\right\} d \xi .
$$

After subtracting the integral equation for $\mathbf{u}$ from the integral equation for $\tilde{\mathbf{u}}$, using (2.10), (2.13), and (2.16), assuming $\mathbf{u}$ and $\tilde{\mathbf{u}} \in C_{M_{1}}^{2}\left(\Omega_{L}, \mathbb{R}^{n}\right)$, one obtains the estimate

$$
\begin{aligned}
\|\mathbf{u}-\tilde{\mathbf{u}}\|_{C_{M_{1}}^{2}} & \leq \frac{n}{c}\left(\left(\frac{\pi}{2}+5\right) M_{3} L+\varepsilon_{0} M_{3}\right)\|\mathbf{u}-\tilde{\mathbf{u}}\|_{C_{M_{1}}^{2}}+\left\|\mathbf{u}_{l}-\tilde{\mathbf{u}}_{l}\right\|_{C_{M_{1}}^{2}} \\
& \leq k\|\mathbf{u}-\tilde{\mathbf{u}}\|_{C_{M_{1}}^{2}}+\left\|\mathbf{u}_{l}-\tilde{\mathbf{u}}_{l}\right\|_{C_{M_{1}}^{2}} \quad \text { with } 0 \leq k<1 .
\end{aligned}
$$


This inequality implies

$$
\|\mathbf{u}-\tilde{\mathbf{u}}\|_{C_{M_{1}}^{2}} \leq \frac{1}{1-k}\left\|\mathbf{u}_{l}-\tilde{\mathbf{u}}_{l}\right\|_{C_{M_{1}}^{2}} \quad \text { with } 0 \leq k<1 .
$$

Since the solution $\mathbf{u}_{l}$ of the linear initial-boundary value problem (2.1)-(2.4) with $\mathbf{F} \equiv \mathbf{0}$ depends continuously on the initial values it follows from this inequality that small differences between the initial values generate small differences between the solutions $\mathbf{u}$ and $\tilde{\mathbf{u}}$ on $\Omega_{L}$. In other words, the solution of the initial-boundary value problem depends continuously on the initial values. The following theorem on the well-posedness of the problem can now be formulated.

Theorem 2.1. Suppose that $\mathbf{u}_{0}$ and $\mathbf{u}_{1}$ and $\mathbf{F}$ satisfy the assumptions (2.6)-(2.8). Then for any $\varepsilon$ satisfying $0<|\varepsilon| \leq \varepsilon_{0} \ll 1$, the nonlinear initial-boundary value problem (2.1)-(2.4) and the equivalent system of nonlinear integral equations (2.10) have the same, unique and twice continuously differentiable solution for $0 \leq x \leq \pi$ and $0 \leq t \leq L|\varepsilon|^{-1}$, in which $L$ is a sufficiently small, positive constant independent of $\varepsilon$. Furthermore, this unique solution depends continuously on the initial values.

3. On the validity of formal approximations. Since the initial-boundary value problem (2.1)-(2.4) contains a small parameter $\varepsilon$, perturbation methods may be applied to construct approximations of the solution. In most perturbation methods for weakly nonlinear problems a function is constructed that satisfies the differential equation(s) and the initial conditions up to some order depending on the small parameter $\varepsilon$. Such a function is usually called a formal approximation. It requires an additional analysis to show that this formal approximation is an asymptotic approximation as $\varepsilon$ tends to zero. Therefore suppose that on $\Omega_{L}$ (given by (2.13)) a twice continuously differentiable function $\mathbf{v}(x, t ; \varepsilon)$ is constructed satisfying

$$
\begin{array}{ll}
\mathbf{v}_{t t}-C \mathbf{v}_{x x}+\varepsilon \mathbf{F}(\mathbf{v} ; \varepsilon)=|\varepsilon|^{m} \mathbf{c}_{1}(x, t ; \varepsilon), & m>1, \\
\mathbf{v}(x, 0 ; \varepsilon)=\mathbf{u}_{0}(x ; \varepsilon)+|\varepsilon|^{m-1} \mathbf{c}_{2}(x ; \varepsilon) \equiv \mathbf{v}_{0}(x ; \varepsilon), & 0<x<\pi, \\
\mathbf{v}_{t}(x, 0 ; \varepsilon)=\mathbf{u}_{1}(x ; \varepsilon)+|\varepsilon|^{m-1} \mathbf{c}_{3}(x ; \varepsilon) \equiv \mathbf{v}_{1}(x ; \varepsilon), & 0<x<\pi, \\
\mathbf{v}(0, t ; \varepsilon)=\mathbf{v}(\pi, t ; \varepsilon)=\mathbf{0}, & 0 \leq t \leq L|\varepsilon|^{-1},
\end{array}
$$

where $\varepsilon, \mathbf{u}_{0}, \mathbf{u}_{1}$, and $\mathbf{F}$ satisfy $(2.5)-(2.8)$ and where $\mathbf{c}_{1}(x, t ; \varepsilon)=\left(c_{11}(x, t ; \varepsilon), c_{12}(x, t ; \varepsilon)\right.$, $\left.\ldots, c_{1 n}(x, t ; \varepsilon)\right)^{\mathrm{T}}, \quad \mathbf{c}_{2}(x ; \varepsilon)=\left(c_{21}(x ; \varepsilon), c_{22}(x ; \varepsilon), \ldots, c_{2 n}(x ; \varepsilon)\right)^{\mathrm{T}}$, and $\mathbf{c}_{3}(x ; \varepsilon)=$ $\left(c_{31}(x ; \varepsilon), c_{32}(x ; \varepsilon), \ldots, c_{3 n}(x ; \varepsilon)\right)^{\mathrm{T}}$ satisfy

$$
c_{1 i}, \frac{\partial c_{1 i}}{\partial x} \in C\left(\Omega_{L} \times\left[-\varepsilon_{0}, \varepsilon_{0}\right], \mathbb{R}\right) \text { for } i=1,2, \ldots, n
$$

with $\mathbf{c}_{1}(0, t ; \varepsilon)=\mathbf{c}_{1}(\pi, t ; \varepsilon)=\mathbf{0}$ for $0 \leq t \leq L|\varepsilon|^{-1}$,

$$
c_{2 i}, \frac{\partial c_{2 i}}{\partial x}, \frac{\partial^{2} c_{2 i}}{\partial x^{2}} \in C\left([0, \pi] \times\left[-\varepsilon_{0}, \varepsilon_{0}\right], \mathbb{R}\right) \text { for } i=1,2, \ldots, n
$$

with $\mathbf{c}_{2}(0 ; \varepsilon)=\mathbf{c}_{2}(\pi ; \varepsilon)=\frac{\partial^{2} \mathbf{c}_{2}(0 ; \varepsilon)}{\partial x^{2}}=\frac{\partial^{2} \mathbf{c}_{2}(\pi ; \varepsilon)}{\partial x^{2}}=\mathbf{0}$, and for $i=1,2, \ldots, n$

$$
c_{3 i}, \frac{\partial c_{3 i}}{\partial x} \in C\left([0, \pi] \times\left[-\varepsilon_{0}, \varepsilon_{0}\right], \mathbb{R}\right) \quad \text { with } \mathbf{c}_{3}(0 ; \varepsilon)=\mathbf{c}_{3}(\pi ; \varepsilon)=\mathbf{0}
$$


Furthermore (for $i=1,2, \ldots, n), c_{1 i}(x, t ; \varepsilon)$ and its derivative with respect to $x$ are supposed to be uniformly bounded for those values of $t$ and $\varepsilon$ under consideration. From theorem 2.1 it follows that the initial-boundary value problem (3.1)-(3.4) has a unique, twice continuously differentiable solution $\mathbf{v}$ on a time scale of $O\left(|\varepsilon|^{-1}\right)$. This initial-boundary value problem can then be transformed into the equivalent system of integral equations

$$
\mathbf{v}(x, t ; \varepsilon)=\frac{\varepsilon}{2} \int_{0}^{t} \int_{0}^{\pi} G(\xi, \tau ; x, t) \tilde{\mathbf{F}}(\mathbf{v}, \varepsilon)(\xi, \tau) d \xi d \tau+\mathbf{v}_{l}(x, t ; \varepsilon),
$$

where $G$ is given by (2.11) and where $\tilde{\mathbf{F}}$ and $\mathbf{v}_{l}$ are, respectively, given by

$$
\tilde{\mathbf{F}}(\mathbf{v} ; \varepsilon)(x, t) \equiv \mathbf{F}(\mathbf{v} ; \varepsilon)(x, t)-|\varepsilon|^{m-1} \mathbf{c}_{1}(x, t ; \varepsilon)
$$

and

$$
\mathbf{v}_{l}(x, t ; \varepsilon)=\frac{1}{2} \int_{0}^{\pi}\left\{\frac{\partial G}{\partial \tau}(\xi, 0 ; x, t) \mathbf{v}_{0}(\xi ; \varepsilon)-G(\xi, 0 ; x, t) \mathbf{v}_{1}(\xi ; \varepsilon)\right\} d \xi .
$$

Now, it will be shown that the formal approximation $\mathbf{v}$ is an asymptotic approximation $($ as $\varepsilon \rightarrow 0)$ of the solution of the initial-boundary value problem $(2.1)-(2.4)$ if $m>1$, that is, it will be proved that

$$
\|\mathbf{u}-\mathbf{v}\|_{C_{M_{1}}^{2}}=O\left(|\varepsilon|^{m-1}\right) \quad \text { as } \varepsilon \rightarrow 0 .
$$

This result implies that

$$
\lim _{\varepsilon \rightarrow 0}\left|u_{i}(x, t ; \varepsilon)-v_{i}(x, t ; \varepsilon)\right|=0 \text { for } i=1,2, \ldots, n \text { and }(x, t) \in \Omega_{L} .
$$

Subtracting the system of integral equations (3.8) from the system of integral equations (2.10), supposing that $\mathbf{v}_{\text {/ }}$ satisfies (2.14) and that $\tilde{\mathbf{F}}$ satisfies (2.15) and (2.16), using (2.13), (2.16), and the fact that $\mathbf{u}, \mathbf{v} \in C_{M_{1}}^{2}\left(\Omega_{L}, \mathbb{R}^{n}\right)$, the following estimate is obtained:

$$
\begin{aligned}
\|\mathbf{u}-\mathbf{v}\|_{C_{\bar{M}_{1}}^{2}} & \leq \frac{n}{c}\left(\left(\frac{\pi}{2}+5\right) M_{3} L+\varepsilon_{0} M_{3}\right)\|\mathbf{u}-\mathbf{v}\|_{C_{\dot{M}_{1}}^{2}}+\|\mathbf{c}\|_{C_{\dot{M}_{1}}^{2}}+\left\|\mathbf{u}_{l}-\mathbf{v}_{l}\right\|_{C_{\dot{M}_{1}}^{2}} \\
& \leq k\|\mathbf{u}-\mathbf{v}\|_{C_{\bar{M}_{1}}^{2}}+\|\mathbf{c}\|_{C_{\bar{M}_{1}}^{2}}+\left\|\mathbf{u}_{l}-\mathbf{v}_{l}\right\|_{C_{\dot{M}_{1}}^{2}},
\end{aligned}
$$

with $0 \leq k<1, c=\min \left(1, c_{11}, c_{22}, \ldots, c_{n n}\right)$ and where $\mathbf{c}$ is given by

$$
\mathbf{c}(x, t ; \varepsilon)=\frac{|\varepsilon|^{m}}{2} \int_{0}^{t} \int_{0}^{\pi} G(\xi, \tau ; x, t) \mathbf{c}_{1}(\xi, \tau ; \varepsilon) d \xi d \tau,
$$

and where $\mathbf{u}_{l}-\mathbf{v}_{l}$ is given by

$$
\mathbf{u}_{l}(x, t ; \varepsilon)-\mathbf{v}_{l}(x, t ; \varepsilon)=-\frac{|\varepsilon|^{m-1}}{2} \int_{0}^{\pi}\left\{\frac{\partial G}{\partial \tau}(\xi, 0 ; x, t) \mathbf{c}_{2}(\xi ; \varepsilon)-G(\xi, 0 ; x, t) \mathbf{c}_{3}(\xi ; \varepsilon)\right\} d \xi .
$$

Hence,

$$
\|\mathbf{u}-\mathbf{v}\|_{C_{M_{1}}^{2}} \leq \frac{1}{1-k}\left\{\|\mathbf{c}\|_{C_{M_{1}}^{2}}+\left\|\mathbf{u}_{l}-\mathbf{v}_{/}\right\|_{C_{M_{1}}^{2}}\right\} \quad \text { with } 0 \leq k<1 .
$$

From the smoothness properties of $\mathbf{c}_{1}, \mathbf{c}_{2}$, and $\mathbf{c}_{3}$ it follows that there exists a constant $K$ independent of $\varepsilon$, such that

$$
\|\mathbf{c}\|_{C_{i, 1}^{2}} \leq \frac{n}{c}\left(\left(\frac{\pi}{2}+5\right) K L+|\varepsilon| K\right)|\varepsilon|^{m-1}
$$


and

$$
\left\|\mathbf{u}_{l}-\mathbf{v}_{l}\right\|_{C_{M_{1}}^{2}} \leq \frac{n}{c}\left(\frac{\pi}{2}+11\right) K|\varepsilon|^{m-1}
$$

So,

$$
\|\mathbf{u}-\mathbf{v}\|_{C_{M_{1}}^{2}} \leq \frac{n|\varepsilon|^{m-1} K}{c(1-k)}\left\{\left(\frac{\pi}{2}+5\right) L+|\varepsilon|+\frac{\pi}{2}+11\right\} .
$$

For $m>1$ this inequality implies the asymptotic validity (as $\varepsilon \rightarrow 0$ ) of the formal approximation $\mathbf{v}$. The following theorem has now been established.

THEOREM 3.1. Let the formal approximation $\mathbf{v}$ satisfy $(3.1)-(3.4)$, where $\varepsilon, \mathbf{u}_{0}, \mathbf{u}_{1}$, and $\mathbf{F}$ are given by (2.5)-(2.8) and where $\mathbf{c}_{1}, \mathbf{c}_{2}$, and $\mathbf{c}_{3}$ satisfy (3.5)-(3.7). Then for $m>1$, the formal approximation $\mathbf{v}$ is an asymptotic approximation (as $\varepsilon \rightarrow 0$ ) of the solution $\mathbf{u}$ of the nonlinear initial-boundary value problem (2.1)-(2.4). The asymptotic approximation $\mathbf{v}$ is valid for those values of the independent variables $x$ and $t$ for which problem $(2.1)-(2.4)$ has been proved well-posed. That is,

$$
\|\mathbf{u}-\mathbf{v}\|_{C_{\bar{w}_{1}}^{2}}=O\left(|\varepsilon|^{m-1}\right), \quad \text { implying }\left|u_{i}(x, t ; \varepsilon)-v_{i}(x, t ; \varepsilon)\right|=O\left(|\varepsilon|^{m-1}\right)
$$

for $i=1,2, \ldots, n$ and $0 \leq x \leq \pi, 0 \leq t \leq L|\varepsilon|^{-1}$, in which $L$ is a sufficiently small, positive constant independent of $\varepsilon$.

4. A model of the galloping oscillations of overhead transmission lines. In this section a model describing the galloping oscillations of overhead transmission lines will be derived. Galloping is a low frequency, large amplitude phenomenon involving an almost purely vertical oscillation of single-conductor lines on which for instance ice has accreted. The frequencies involved are so low that the assumption can be made that the aerodynamic forces are as in steady flow. Another consequence of these low frequencies is that structural damping may be neglected. In severe cases galloping may give rise to conductor damage due to impact of conductor lines and due to flashover as a result of a phase difference between conductor lines, for which the mutual distance has become too small. The usual conditions (see [15]) causing galloping are those of incipient icing in a stable atmospheric environment implying uniform (but not necessarily high velocity) airflows.

In [1] an oscillator with two degrees of freedom has been considered to describe the oscillations of a rigid circular cylinder with a small ice ridge. In that approach a system of two coupled, ordinary differential equations is obtained, describing the displacements of the cylinder in two directions. In [7] a cylinder-shaped transmission line has been considered to describe the vertical displacement of the conductor due to galloping. In this section the vertical as well as the horizontal displacements of the transmission line will be taken into account. To describe the galloping oscillations a circular cylinder-shaped conductor will be considered to be situated in a horizontal airflow. Such a symmetric circular conductor cannot exhibit galloping because there cannot be generated a force that lifts the conductor against gravity. On the other hand, a conductor on which ice has accreted may gallop if it adopts a suitable attitude to the wind. To describe this phenomenon a right-handed coordinate system is set up where one of the endpoints of the conductor is the origin. Through this point 
three mutually perpendicular axes (the $x-, y$-, and $z$-axes) are drawn, where the $z$ axis coincides with the direction of gravity and the $y$-axis with the direction of the airflow. The three coordinate axes span the three coordinate planes in space, the $(x, y)-,(x, z)$, and $(y, z)$-planes. On each coordinate axis a unit vector is fixed: on the $x$-axis the vector $\mathbf{e}_{x}$, on the $y$-axis the vector $\mathbf{e}_{y}$, and on the $z$-axis the vector $\mathbf{e}_{z}$, which has a direction opposite to gravity. The coordinate axes are directed by these vectors, such that a right-handed coordinate system is obtained. The coordinates of the endpoints of the conductor are supposed to be $(0,0,0)$ and $(l, 0,0)$, where $l$ is the distance between the endpoints. To model galloping, a cross section (perpendicular to the $x$-axis) of the conductor with ice ridge is considered. Assume that all crosssectional shapes are congruent and have only one axis of symmetry. Along the axis of symmetry of a cross section a unit vector $\mathbf{e}_{s}$ is defined to be directing away from the ice ridge and starting in the center of the uniced cross section. In Fig. 4.1 the center of the cross section is considered to be at $x=x_{0}, y=y_{0}$, and $z=z_{0}$ with $0<x_{0}<l \leq l_{c}$, where $l_{c}$ is the length of the conductor.

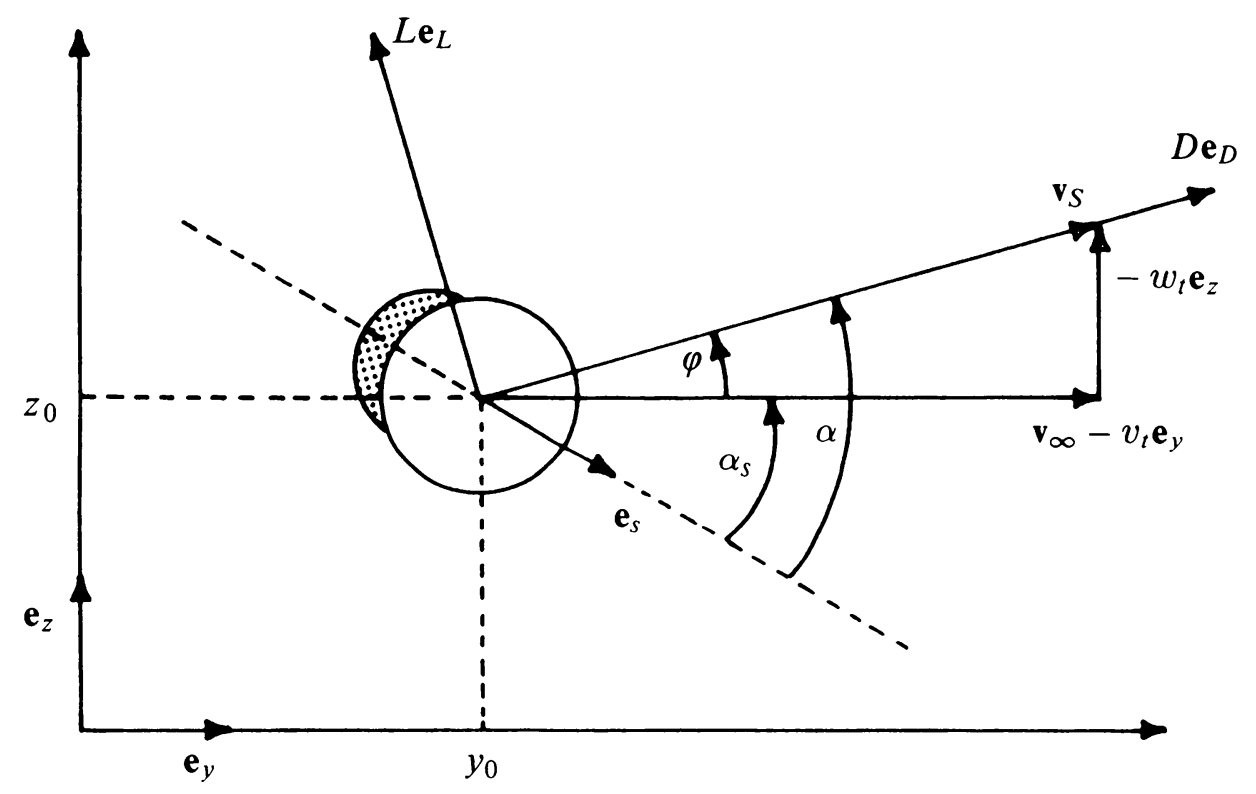

Fig. 4.1. Cross section of the circular cylinder-shaped conductor with ice ridge in a uniform airflow $\mathbf{v}_{\infty}=v_{\infty} \mathbf{e}_{y}$.

At $x=x_{0}$ and time $t$ the $y$-coordinate and the $z$-coordinate of the center of the cross section are denoted by $v\left(x_{0}, t\right)$ and $w\left(x_{0}, t\right)$, respectively. Assume that every cross section perpendicular to the $x$-axis oscillates in the $(y, z)$-plane. Furthermore, the torsion of the conductor is not taken into account. Let the static angle of attack $\alpha_{s}$ (assumed to be constant and identical for all cross sections) be the angle between $\mathbf{e}_{s}$ and the uniform airflow $\mathbf{v}_{\infty}=v_{\infty} \mathbf{e}_{y}\left(v_{\infty}>0\right)$, that is, $\alpha_{s}:=L\left(\mathbf{e}_{s}, \mathbf{v}_{\infty}\right)$ with $\left|\alpha_{s}\right| \leq \pi$. In this uniform airflow with flow velocity $\mathbf{v}_{\infty}=v_{\infty} \mathbf{e}_{y}$ the conductor may oscillate due to the lift force $L \mathbf{e}_{L}$ and the drag force $D \mathbf{e}_{D}$. It should be noted that the drag 
force $D \mathbf{e}_{D}$ has the direction of the virtual wind velocity $\mathbf{v}_{s} \equiv \mathbf{v}_{\infty}-v_{t} \mathbf{e}_{y}-w_{t} \mathbf{e}_{z}$ and that the lift force $L \mathbf{e}_{L}$ has a direction perpendicular to the virtual wind velocity $\mathbf{v}_{s}$ $\left(\mathbf{e}_{L}\right.$ is chosen perpendicular and anti-clockwise to $\mathbf{e}_{D}$ ). In Fig. 4.1 the forces $L \mathbf{e}_{L}$ and $D \mathbf{e}_{D}$ acting on the cross section are given. Now the conductor is considered to be a one-dimensional continuum in which the only interaction between different parts is due to a tension $T$, which is assumed to be constant in space and time. The validity of this assumption will be discussed in Sec. 6 . The equations describing the horizontal and the vertical motion of the conductor are then given by

$$
\begin{aligned}
& \rho_{c} A v_{t t}-T A \frac{\partial}{\partial x}\left(\frac{v_{x}}{\left(1+v_{x}^{2}+w_{x}^{2}\right)^{1 / 2}}\right)=D \cos \phi-L \sin \phi, \\
& \rho_{c} A w_{t t}-T A \frac{\partial}{\partial x}\left(\frac{w_{x}}{\left(1+v_{x}^{2}+w_{x}^{2}\right)^{1 / 2}}\right)=-\rho_{c} A g+D \sin \phi+L \cos \phi,
\end{aligned}
$$

where $D$ and $L$ are the magnitudes of the drag and lift force acting on the conductor per unit length of the conductor, respectively, $\rho_{c}$ is the mass-density of the conductor (including the small ice ridge), $A$ is the constant cross-sectional area of the conductor (including the small ice ridge), $\phi$ is the angle between $\mathbf{v}_{\infty}-v_{t} \mathbf{e}_{y}$ and $\mathbf{v}_{s}$ (that is, $\phi:=\angle\left(\mathbf{v}_{\infty}-v_{t} \mathbf{e}_{y}, \mathbf{v}_{s}\right)$ with $\left.|\phi| \leq \pi\right)$, and $g$ is the gravitational acceleration. The magnitudes $D$ and $L$ of the aerodynamic forces may be given by

$$
\begin{aligned}
& D=\frac{1}{2} \rho_{a} d c_{D}(\alpha) v_{s}^{2}, \\
& L=\frac{1}{2} \rho_{a} d c_{L}(\alpha) v_{s}^{2},
\end{aligned}
$$

where $\rho_{a}$ is the air density, $d$ is the diameter of the cross section of the uniced conductor, $v_{s}^{2}=\left(v_{\infty}-v_{t}\right)^{2}+w_{t}^{2}, \alpha$ is the angle between $\mathbf{e}_{s}$ and $\mathbf{v}_{s}$ (that is, $\alpha:=\angle\left(\mathbf{e}_{s}, \mathbf{v}_{s}\right)$ with $|\alpha| \leq \pi)$, and $c_{D}(\alpha)$ and $c_{L}(\alpha)$ are the quasi-steady drag- and lift-coefficients, which may be obtained from wind-tunnel measurements. For a certain range of values of $v_{\infty}$ some characteristic results from wind-tunnel experiments are given in Fig. 4.2 (see also $[1,7,13]$ ).

According to the den Hartog criterion [5] a two-dimensional section is aerodynamically unstable if

$$
c_{D}(\alpha)+\frac{d c_{L}(\alpha)}{d \alpha}<0
$$

From Fig. 4.2 it follows that this condition is likely to be satisfied for some interval in $\alpha$ with $\alpha_{0}<\alpha<\alpha_{2}$, where $\alpha_{0}$ and $\alpha_{2}$ are determined by $c_{D}(\alpha)+d c_{L}(\alpha) / d \alpha=0$. For a certain range of values of $\alpha$ (including those values which satisfy the den Hartog criterion) the drag- and lift-coefficients $c_{D}(\alpha)$ and $c_{L}(\alpha)$ can be approximated by

$$
\begin{gathered}
c_{D 0}+c_{D 1}\left(\alpha-\alpha_{1}\right)+c_{D 2}\left(\alpha-\alpha_{1}\right)^{2}+c_{D 3}\left(\alpha-\alpha_{1}\right)^{3}, \\
c_{L 1}\left(\alpha-\alpha_{1}\right)+c_{L 2}\left(\alpha-\alpha_{1}\right)^{2}+c_{L 3}\left(\alpha-\alpha_{1}\right)^{3},
\end{gathered}
$$

with $c_{D 0}>0, c_{L 1}<0, c_{L 3}>0, \alpha_{0}<\alpha_{1}<\alpha_{2}$ where $c_{L}\left(\alpha_{1}\right)=0$. Since galloping is a low-frequency phenomenon it may be assumed that $\left|v_{t}\right| \ll v_{\infty}$ and $\left|w_{t}\right| \ll v_{\infty}$. The right-hand sides of Eq. (4.1) and (4.2) can be considered as functions of $v_{t} / v_{\infty}$ and $w_{t} / v_{\infty}$, and so these right-hand sides are to be expanded in Taylor series near $v_{t} / v_{\infty}=0$ and $w_{t} / v_{\infty}=0$. To obtain these Taylor series the approximations (4.5) 


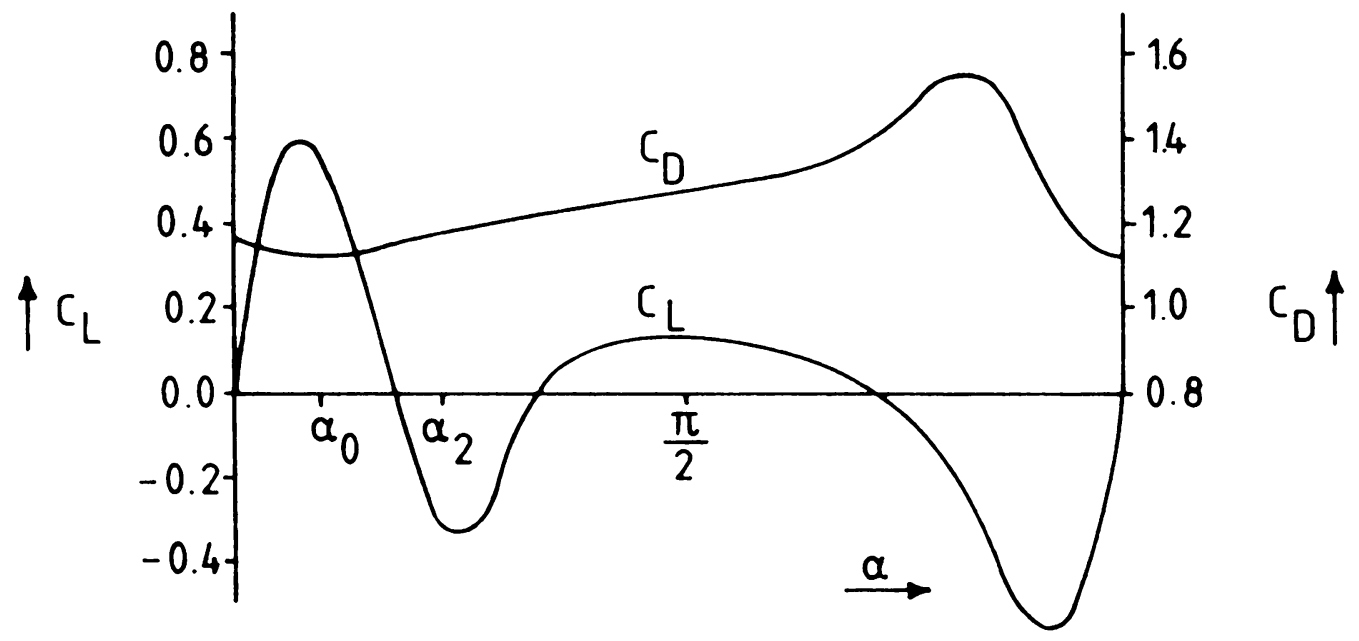

Fig. 4.2. Typical variation of the drag- and lift-coefficients $c_{D}$ and $c_{L}$ with angle of attack for a symmetric profile with small icy nose.

and (4.6) for $c_{D}$ and $c_{L}$, respectively, are used. Since the amplitudes of the oscillations are small compared to the length of the conductor and since galloping is an oscillation described by the lower modes of vibration, it may be assumed that $\left|v_{x}\right| \ll 1$ and $\left|w_{x}\right| \ll 1$. So, the left-hand sides of Eqs. (4.1) and (4.2) are expanded in Taylor series near $v_{x}=0$ and $w_{x}=0$. Neglecting terms of degree four and higher, Eqs. (4.1) and (4.2) become

$$
\begin{aligned}
& v_{t t}-c^{2}\left\{\left(1-\frac{3}{2} v_{x}^{2}-\frac{1}{2} w_{x}^{2}\right) v_{x x}-v_{x} w_{x} w_{x x}\right\} \\
& =\frac{\rho_{a} d v_{\infty}^{2}}{2 \rho_{c} A}\left\{a_{00}+a_{10} \frac{v_{t}}{v_{\infty}}+a_{01} \frac{w_{t}}{v_{\infty}}+a_{20} \frac{v_{t}^{2}}{v_{\infty}^{2}}+a_{11} \frac{v_{t} w_{t}}{v_{\infty}^{2}}+a_{02} \frac{w_{t}^{2}}{v_{\infty}^{2}}+a_{03} \frac{w_{t}^{3}}{v_{\infty}^{3}}\right\}, \\
& w_{t t}-c^{2}\left\{-v_{x} w_{x} v_{x x}+\left(1-\frac{1}{2} v_{x}^{2}-\frac{3}{2} w_{x}^{2}\right) w_{x x}\right\} \\
& =\frac{\rho_{a} d v_{\infty}^{2}}{2 \rho_{c} A}\left\{b_{00}+b_{10} \frac{v_{t}}{v_{\infty}}+b_{01} \frac{w_{t}}{v_{\infty}}+b_{20} \frac{v_{t}^{2}}{v_{\infty}^{2}}+b_{11} \frac{v_{t} w_{t}}{v_{\infty}^{2}}+b_{02} \frac{w_{t}^{2}}{v_{\infty}^{2}}+b_{03} \frac{w_{t}^{3}}{v_{\infty}^{3}}\right\},
\end{aligned}
$$

where $c=\left(T \rho_{c}^{-1}\right)^{1 / 2}$,

$$
\begin{array}{ll}
a_{00}=c_{D}\left(\alpha_{s}\right), & a_{10}=-2 c_{D}\left(\alpha_{s}\right), \\
a_{01}=c_{L}\left(\alpha_{s}\right)-\frac{d c_{D}\left(\alpha_{s}\right)}{d \alpha}, & a_{20}=c_{D}\left(\alpha_{s}\right), \\
a_{11}=-c_{L}\left(\alpha_{s}\right)+\frac{d c_{D}\left(\alpha_{s}\right)}{d \alpha}, & a_{02}=\frac{1}{2} c_{D}\left(\alpha_{s}\right)-\frac{d c_{L}\left(\alpha_{s}\right)}{d \alpha}+\frac{1}{2} \frac{d^{2} c_{D}\left(\alpha_{s}\right)}{d \alpha^{2}}, \\
a_{03}=\frac{1}{2} c_{L}\left(\alpha_{S}\right)-\frac{1}{6} \frac{d c_{D}\left(\alpha_{s}\right)}{d \alpha}+\frac{1}{2} \frac{d^{2} c_{L}\left(\alpha_{s}\right)}{d \alpha^{2}}-\frac{1}{6} \frac{d^{3} c_{D}\left(\alpha_{s}\right)}{d \alpha^{3}}
\end{array}
$$




$$
\begin{array}{ll}
b_{00}=c_{L}\left(\alpha_{s}\right)-\frac{2 \rho_{c} A g}{\rho_{a} d v_{\infty}^{2}}, & b_{10}=-2 c_{L}\left(\alpha_{s}\right), \\
b_{01}=-c_{D}\left(\alpha_{s}\right)-\frac{d c_{L}\left(\alpha_{s}\right)}{d \alpha}, & b_{20}=c_{L}\left(\alpha_{s}\right), \\
b_{11}=c_{D}\left(\alpha_{s}\right)+\frac{d c_{L}\left(\alpha_{s}\right)}{d \alpha}, & b_{02}=\frac{1}{2} c_{L}\left(\alpha_{s}\right)+\frac{d c_{D}\left(\alpha_{s}\right)}{d \alpha}+\frac{1}{2} \frac{d^{2} c_{L}\left(\alpha_{s}\right)}{d \alpha^{2}}, \\
b_{03}=-\frac{1}{2} c_{D}\left(\alpha_{s}\right)-\frac{1}{6} \frac{d c_{L}\left(\alpha_{s}\right)}{d \alpha}-\frac{1}{2} \frac{d^{2} c_{D}\left(\alpha_{s}\right)}{d \alpha^{2}}-\frac{1}{6} \frac{d^{3} c_{L}\left(\alpha_{s}\right)}{d \alpha^{3}} .
\end{array}
$$

In (4.9) the approximations (4.5) and (4.6) for $c_{D}$ and $c_{L}$, respectively, should be substituted. Applying the transformations

$$
v(x, t)=\tilde{v}(x, t)-\frac{\rho_{a} d v_{\infty}^{2} a_{00}}{4 T A} x(x-l)
$$

and

$$
w(x, t)=\tilde{w}(x, t)-\frac{\rho_{a} d v_{\infty}^{2} b_{00}}{4 T A} x(x-l)
$$

and using the dimensionless variables

$$
\bar{v}=\frac{\pi c}{l v_{\infty}} \tilde{v}, \quad \bar{w}=\frac{\pi c}{l v_{\infty}} \tilde{w}, \quad \bar{x}=\frac{\pi}{l} x, \quad \text { and } \quad \bar{t}=\frac{\pi c}{l} t,
$$

Eqs. (4.7) and (4.8) become

$$
\begin{aligned}
& \bar{v}_{\bar{t} \bar{t}}-\bar{v}_{\bar{x} \bar{x}}+\left(\frac{v_{\infty}}{c}\right)^{2}\left\{\left(\frac{3}{2}\left(\bar{v}_{\bar{x}}-\frac{\rho_{a} d v_{\infty} l a_{00}}{4 \pi \rho_{c} A c}(2 \bar{x}-\pi)\right)^{2}\right.\right. \\
&+\left.\frac{1}{2}\left(\bar{w}_{\bar{x}}-\frac{\rho_{a} d v_{\infty} l b_{00}}{4 \pi \rho_{c} A c}(2 \bar{x}-\pi)\right)^{2}\right)\left(\bar{v}_{\bar{x} \bar{x}}-\frac{\rho_{a} d v_{\infty} l a_{00}}{2 \pi \rho_{c} A c}\right) \\
&+\left(\bar{v}_{\bar{x}}-\frac{\rho_{a} d v_{\infty} l a_{00}}{4 \pi \rho_{c} A c}(2 \bar{x}-\pi)\right)\left(\bar{w}_{\bar{x}}-\frac{\rho_{a} d v_{\infty} l b_{00}}{4 \pi \rho_{c} A c}(2 \bar{x}-\pi)\right) \\
&\left.\times\left(\bar{w}_{\bar{x} \bar{x}}-\frac{\rho_{a} d v_{\infty} l b_{00}}{2 \pi \rho_{c} A c}\right)\right\} \\
&= \frac{\rho_{a} d l}{2 \pi \rho_{c} A}\left(\frac{v_{\infty}}{c}\right)\left\{a_{10} \bar{v}_{\bar{t}}+a_{01} \bar{w}_{\bar{t}}+a_{20} \bar{v}_{\bar{t}}^{2}+a_{11} \bar{v}_{\bar{t}} \bar{w}_{\bar{t}}+a_{02} \bar{w}_{\bar{t}}^{2}+a_{03} \bar{w}_{\bar{t}}^{3}\right\} \\
& \bar{w}_{\bar{t} \bar{t}}-\bar{w}_{\bar{x} \bar{x}}+\left(\frac{v_{\infty}}{c}\right)^{2}\left\{\left(\bar{v}_{\bar{x}}-\frac{\rho_{a} d v_{\infty} l a_{00}}{4 \pi \rho_{c} A c}(2 \bar{x}-\pi)\right)\left(\bar{w}_{\bar{x}}-\frac{\rho_{a} d v_{\infty} l b_{00}}{4 \pi \rho_{c} A c}(2 \bar{x}-\pi)\right)\right. \\
& \times\left(\bar{v}_{\bar{x} \bar{x}}-\frac{\rho_{a} d v_{\infty} l a_{00}}{2 \pi \rho_{c} A c}\right)+\left(\frac{1}{2}\left(\bar{v}_{\bar{x}}-\frac{\rho_{a} d v_{\infty} l a_{00}}{4 \pi \rho_{c} A c}(2 \bar{x}-\pi)\right)^{2}\right. \\
&\left.\left.+\frac{3}{2}\left(\bar{w}_{\bar{x}}-\frac{\rho_{a} d v_{\infty} l b_{00}}{4 \pi \rho_{c} A c}(2 \bar{x}-\pi)\right)^{2}\right)\left(\bar{w}_{\bar{x} \bar{x}}-\frac{\rho_{a} d v_{\infty} l b_{00}}{2 \pi \rho_{c} A c}\right)\right\} \\
&=\frac{\rho_{a} d l}{2 \pi \rho_{c} A}\left(\frac{v_{\infty}}{c}\right)\left\{b_{10} \bar{v}_{\bar{t}}+b_{01} \bar{w}_{\bar{t}}+b_{20} \bar{v}_{\bar{t}}^{2}+b_{11} \bar{v}_{\bar{t}} \bar{w}_{\bar{t}}+b_{02} \bar{w}_{\bar{t}}^{2}+b_{03} \bar{w}_{\bar{t}}^{3}\right\}
\end{aligned}
$$


where the dimensionless constants $a_{00}, a_{10}, \ldots, b_{03}$ are given by (4.9). Typical values of the physical quantities in a practical application are: $l=400 \mathrm{~m}, d=0.04 \mathrm{~m}$, $A=\pi(d / 2)^{2}=4 \pi \cdot 10^{-4} \mathrm{~m}^{2}, \rho_{c}=4000 \mathrm{~kg} / \mathrm{m}^{3}, \rho_{a}=1.25 \mathrm{~kg} / \mathrm{m}^{3}, g=10 \mathrm{~m} / \mathrm{s}^{2}$, and $v_{\infty}=10 \mathrm{~m} / \mathrm{s}$. The tension $T$ in the conductor is estimated by $\frac{1}{2} \rho_{c} g(l / 2)^{2} s_{0}^{-1}$, where $s_{0}$ (usually 2 or 3 percent of $l$ ) is the sag of the conductor. Let $s_{0}$ be $10 \mathrm{~m}$; then $T=8 \times 10^{7} \mathrm{~kg} / \mathrm{ms}^{2}$ and consequently $c=140 \mathrm{~m} / \mathrm{s}(c$ may be identified with the speed of propagation of transversal waves in the conductor). By putting $\tilde{\varepsilon}=v_{\infty} / c$ and by assuming that the static angle of attack $\alpha_{s}$ is such that galloping may set in according to the instability criterion of den Hartog [5], that is, by assuming that $\alpha_{s}=\alpha_{1}+O(\tilde{\varepsilon})$, it then follows that $\left(a_{00} \approx \frac{12}{10}\right)$

$$
\tilde{\varepsilon}=\frac{v_{\infty}}{c} \approx \frac{1}{14}, \quad \frac{\rho_{a} d l}{2 \pi \rho_{c} A} \approx \frac{5}{8}, \quad \frac{\rho_{a} d v_{\infty} l a_{00}}{4 \pi \rho_{c} A c} \approx \frac{1}{37}, \quad \text { and } \quad \frac{\rho_{a} d v_{\infty} l b_{00}}{4 \pi \rho_{c} A c} \approx \frac{5}{11}
$$

Equations (4.10) and (4.11) now become, up to order $\tilde{\varepsilon}$,

$$
\begin{gathered}
\bar{v}_{\bar{t} \bar{t}}-\bar{v}_{\bar{x} \bar{x}}=\varepsilon\left\{a_{10} \bar{v}_{\bar{t}}+a_{01} \bar{w}_{\bar{t}}+a_{20} \bar{v}_{\bar{t}}^{2}+a_{11} \bar{v}_{\bar{t}} \bar{w}_{\bar{t}}+a_{02} \bar{w}_{\bar{t}}^{2}+a_{03} \bar{w}_{\bar{t}}^{3}\right\}, \\
\bar{w}_{\bar{t} \bar{t}}-\bar{w}_{\bar{x} \bar{x}}=\varepsilon\left\{b_{10} \bar{v}_{\bar{t}}+b_{01} \bar{w}_{\bar{t}}+b_{20} \bar{v}_{\bar{t}}^{2}+b_{11} \bar{v}_{\bar{t}} \bar{w}_{\bar{t}}+b_{02} \bar{w}_{\bar{t}}^{2}+b_{03} \bar{w}_{\bar{t}}^{3}\right\},
\end{gathered}
$$

where $\varepsilon=\frac{\rho_{a} d l}{2 \pi \rho_{c} A} \tilde{\varepsilon}$ is a small, positive parameter and where the constants $a_{10}, a_{01}$, $\ldots, b_{03}$ are given by (4.9) with $\alpha_{s}=\alpha_{1}$, that is, $a_{10}=-2 c_{D 0}, a_{01}=-c_{D 1}, a_{20}=c_{D 0}$, $a_{11}=c_{D 1}, a_{02}=\frac{1}{2} c_{D 0}+c_{D 2}-c_{L 1}, a_{03}=-\frac{1}{6} c_{D 1}-c_{D 3}+c_{L 2}, b_{10}=0, b_{01}=-c_{D 0}-c_{L 1}$, $b_{20}=0, b_{11}=c_{D 0}+c_{L 1}, b_{02}=c_{D 1}+c_{L 2}$, and $b_{03}=-\frac{1}{2} c_{D 0}-c_{D 2}-\frac{1}{6} c_{L 1}-c_{L 3}$. For the cross-sectional shape of the conductor with small ice ridge under consideration the aerodynamic coefficients $c_{D 0}, c_{D 1}, c_{D 2}, c_{D 3}, c_{L 1}, c_{L 2}$, and $c_{L 3}$ may be determined from wind-tunnel measurements (as for instance given in Fig. 4.2). Figure 4.2 suggests that $c_{D 0}>0, c_{D 1}>0, c_{L 1}<0, c_{L 3}>0, c_{D 0}+c_{L 1}<0$, and $\frac{1}{2} c_{D 0}+c_{D 2}+\frac{1}{6} c_{L 1}+c_{L 3}>0$.

If a conductor with fixed endpoints is considered, the boundary conditions $\bar{v}(0, \bar{t})$ $=\bar{v}(\pi, \bar{t})=\bar{w}(0, \bar{t})=\bar{w}(\pi, \bar{t})=0$ are obtained. In the next section the partial differential equations (4.12) and (4.13) subject to these Dirichlet boundary conditions and the initial values $\bar{v}(\bar{x}, 0)=\bar{v}_{0}(\bar{x}), \bar{v}_{\bar{t}}(\bar{x}, 0)=\bar{v}_{1}(\bar{x}), \bar{w}(\bar{x}, 0)=\bar{w}_{0}(\bar{x})$, and $\bar{w}_{\bar{t}}(\bar{x}, 0)=\bar{w}_{1}(\bar{x})$ will be studied, where $\bar{v}_{0}$ and $\bar{w}_{0}$ can be regarded as the initial displacement of the conductor in the $y$-and $z$-directions, respectively, and where $\bar{v}_{1}$ and $\bar{w}_{1}$ represent the initial velocity of the conductor in the $y$-and $z$-directions, respectively.

5. An asymptotic approximation of the solution of a system of nonlinear wave equations. In this section the following initial-boundary value problem for a twice continuously differentiable and vector-valued function $\mathbf{u}(x, t ; \varepsilon)=(\bar{v}(x, t ; \varepsilon), \bar{w}(x, t ; \varepsilon))^{\mathrm{T}}$ 
will be considered:

$$
\begin{aligned}
\bar{v}_{t t}-\bar{v}_{x x}=\varepsilon\left(a_{10} \bar{v}_{t}+a_{01} \bar{w}_{t}+a_{20} \bar{v}_{t}^{2}+a_{11} \bar{v}_{t} \bar{w}_{t}+a_{02} \bar{w}_{t}^{2}+a_{03} \bar{w}_{t}^{3}\right), & \\
\bar{w}_{t t}-\bar{w}_{x x}=\varepsilon\left(b_{01} \bar{w}_{t}+b_{11} \bar{v}_{t} \bar{w}_{t}+b_{02} \bar{w}_{t}^{2}+b_{03} \bar{w}_{t}^{3}\right), & 0<x<\pi, t>0 \\
\bar{v}(x, 0 ; \varepsilon)=\bar{v}_{0}(x ; \varepsilon) \equiv\left(V_{00}+\varepsilon V_{01}\right) \sin (m x), & 0<x<\pi, t>0, \\
\bar{w}(x, 0 ; \varepsilon)=\bar{w}_{0}(x ; \varepsilon) \equiv\left(W_{00}+\varepsilon W_{01}\right) \sin (n x), & 0<x<\pi, \\
\bar{v}_{t}(x, 0 ; \varepsilon)=\bar{v}_{1}(x ; \varepsilon) \equiv\left(V_{10}+\varepsilon V_{11}\right) \sin (m x), & 0<x<\pi \\
\bar{w}_{t}(x, 0 ; \varepsilon)=\bar{w}_{1}(x ; \varepsilon) \equiv\left(W_{10}+\varepsilon W_{11}\right) \sin (n x), & 0<x<\pi, \\
\mathbf{u}(0, t ; \varepsilon) & =\mathbf{u}(\pi, t ; \varepsilon)=\mathbf{0}, \quad t \geq 0,
\end{aligned}
$$

where $a_{10}, a_{01}, \ldots, b_{03}, V_{00}, V_{01}, \ldots, W_{10}, W_{11}$ are constants independent of $\varepsilon, m$ and $n$ are integers, and $0<\varepsilon \ll 1$. From Theorem 2.1 it follows that this initial-boundary value problem is well-posed on $\Omega_{L}$ (given by (2.13)).

For arbitrary $m, n, a_{10}, a_{01}, \ldots, b_{03}, V_{00}, \ldots, W_{10}$, and $W_{11}$, an asymptotic approximation (as $\varepsilon$ tends to zero) of the solution of (5.1)-(5.7) will be constructed in this section. In view of computational difficulties (as also has been noticed in [10]), whenever one assumes an infinite series representation for the solution of the nonlinear initial-boundary value problem, one may alternatively investigate the problem in the characteristic coordinates $\sigma=x-t$ and $\xi=x+t$. In this approach the initialboundary value problem (5.1)-(5.7) has to be replaced by an initial-value problem. This replacement requires one to extend in $x$ to odd and $2 \pi$-periodic functions the dependent variable $\mathbf{u}(x, t)$, the right-hand sides of Eqs. (5.1) and (5.2) as well as the functions $\bar{v}_{0}, \bar{v}_{1}, \bar{w}_{0}$, and $\bar{w}_{1}$. For simplicity the extended functions $\mathbf{u}, \bar{v}_{0}, \bar{v}_{1}, \bar{w}_{0}$, and $\bar{w}_{1}$ will be denoted by the same symbols. In constructing an approximation of the solution $\mathbf{u}(x, t)=\mathbf{u}^{*}(\sigma, \xi)$ of this initial-value problem, a two-time-scales perturbation method will be used, because the straightforward perturbation expansion $\mathbf{u}_{0}^{*}(\sigma, \xi)+\varepsilon \mathbf{u}_{1}^{*}(\sigma, \xi)+\cdots$ causes secular terms. Applying the two-time-scales perturbation method, $\mathbf{u}(x, t)$ is supposed to be a function of $\sigma=x-t, \xi=x+t$, and $\tau=\varepsilon t$. By putting $\mathbf{u}(x, t)=\tilde{\mathbf{u}}(\sigma, \xi, \tau)=(\tilde{v}(\sigma, \xi, \tau), \tilde{w}(\sigma, \xi, \tau))^{\mathrm{T}}$ the following initialvalue problem is obtained:

$$
\begin{gathered}
-4 \tilde{v}_{\sigma \xi}+2 \varepsilon\left(\tilde{v}_{\xi \tau}-\tilde{v}_{\sigma \tau}\right)+\varepsilon^{2} \tilde{v}_{\tau \tau}=\varepsilon\left(a_{10}\left(-\tilde{v}_{\sigma}+\tilde{v}_{\xi}+\varepsilon \tilde{v}_{\tau}\right)+a_{01}\left(-\tilde{w}_{\sigma}+\tilde{w}_{\xi}+\varepsilon \tilde{w}_{\tau}\right)\right. \\
\left.+p_{1}\left(\sigma+\xi,-\tilde{v}_{\sigma}+\tilde{v}_{\xi}+\varepsilon \tilde{v}_{\tau},-\tilde{w}_{\sigma}+\tilde{w}_{\xi}+\varepsilon \tilde{w}_{\tau}\right)+a_{03}\left(-\tilde{w}_{\sigma}+\tilde{w}_{\xi}+\varepsilon \tilde{w}_{\tau}\right)^{3}\right), \\
\text { for }-\infty<\sigma<\xi<\infty, \tau>0, \\
-4 \tilde{w}_{\sigma \xi}+2 \varepsilon\left(\tilde{w}_{\xi \tau}-\tilde{w}_{\sigma \tau}\right)+\varepsilon^{2} \tilde{w}_{\tau \tau}=\varepsilon\left(b_{01}\left(-\tilde{w}_{\sigma}+\tilde{w}_{\xi}+\varepsilon \tilde{w}_{\tau}\right)\right. \\
\left.+p_{2}\left(\sigma+\xi,-\tilde{v}_{\sigma}+\tilde{v}_{\xi}+\varepsilon \tilde{v}_{\tau},-\tilde{w}_{\sigma}+\tilde{w}_{\xi}+\varepsilon \tilde{w}_{\tau}\right)+b_{03}\left(-\tilde{w}_{\sigma}+\tilde{w}_{\xi}+\varepsilon \tilde{w}_{\tau}\right)^{3}\right), \\
\text { for }-\infty<\sigma<\xi<\infty, \tau>0
\end{gathered}
$$

$$
\begin{aligned}
\tilde{v}(\sigma, \xi, \tau) & =\left(V_{00}+\varepsilon V_{01}\right) \sin (m \sigma), \text { for }-\infty<\sigma=\xi<\infty, \tau=0, \\
\tilde{w}(\sigma, \xi, \tau) & =\left(W_{00}+\varepsilon W_{01}\right) \sin (n \sigma), \text { for }-\infty<\sigma=\xi<\infty, \tau=0
\end{aligned}
$$




$$
\begin{array}{r}
-\tilde{v}_{\sigma}(\sigma, \xi, \tau)+\tilde{v}_{\xi}(\sigma, \xi, \tau)+\varepsilon \tilde{v}_{\tau}(\sigma, \xi, \tau)=v_{1}(\sigma)=\left(V_{10}+\varepsilon V_{11}\right) \sin (m \sigma), \\
\text { for }-\infty<\sigma=\xi<\infty, \tau=0, \\
-\tilde{w}_{\sigma}(\sigma, \xi, \tau)+\tilde{w}_{\xi}(\sigma, \xi, \tau)+\varepsilon \tilde{w}_{\tau}(\sigma, \xi, \tau)=w_{1}(\sigma)=\left(W_{10}+\varepsilon W_{11}\right) \sin (n \sigma), \\
\text { for }-\infty<\sigma=\xi<\infty, \tau=0,
\end{array}
$$

where $p_{1}(a, b, c)=E(a / 2)\left\{a_{20} b^{2}+a_{11} b c+a_{02} c^{2}\right\}$ and $p_{2}(a, b, c)=E(a / 2)\left\{b_{11} b c+\right.$ $\left.b_{02} c^{2}\right\}$ with $E(x)=1$ for $0<x<\pi, E(x)=-1$ for $-\pi<x<0, E(0)=E(\pi)=0$, and $E(x)$ is $2 \pi$-periodic in $x$. Furthermore, $\tilde{v}=\tilde{w}=0$ if $\sigma=k \pi-\theta, \xi=k \pi+\theta$, and $\tau=\varepsilon \theta$ with $k \in \mathbb{Z}$ and $\theta \geq 0$. Now it is assumed that $\tilde{v}$, respectively $\tilde{w}$, may be approximated by the formal perturbation expansions $v_{0}(\sigma, \xi, \tau)+\varepsilon v_{1}(\sigma, \xi, \tau)+$ $\varepsilon^{2} v_{2}(\sigma, \xi, \tau)+\cdots$ and $w_{0}(\sigma, \xi, \tau)+\varepsilon w_{1}(\sigma, \xi, \tau)+\varepsilon^{2} w_{2}(\sigma, \xi, \tau)+\cdots$, respectively. By substituting these expansions into (5.8)-(5.13), and after equating the coefficients of like powers in $\varepsilon$, it follows from the powers 0 and 1 of $\varepsilon$ that $v_{0}$ and $w_{0}$ should satisfy

$$
\begin{aligned}
-4 v_{0_{\sigma \xi} \xi} & =0, & & -\infty<\sigma<\xi<\infty, \tau>0,(5.14) \\
-4 w_{0_{\sigma \xi}} & =0, & & -\infty<\sigma<\xi<\infty, \tau>0,(5.15) \\
v_{0}(\sigma, \xi, \tau) & =V_{00} \sin (m \sigma), & & -\infty<\sigma=\xi<\infty, \tau=0,(5.16) \\
w_{0}(\sigma, \xi, \tau) & =W_{00} \sin (n \sigma), & & -\infty<\sigma=\xi<\infty, \tau=0,(5.17) \\
-v_{0_{\sigma}}(\sigma, \xi, \tau)+v_{0_{\xi}}(\sigma, \beta, \tau) & =V_{10} \sin (m \sigma), & & -\infty<\sigma=\xi<\infty, \tau=0,(5.18) \\
-w_{0_{\sigma}}(\sigma, \xi, \tau)+w_{0_{\xi}}(\sigma, \xi, \tau) & =W_{10} \sin (n \sigma), & & -\infty<\sigma=\xi<\infty, \tau=0,(5.19)
\end{aligned}
$$

and that $v_{1}$ and $w_{1}$ should satisfy

$$
\begin{gathered}
-4 v_{1_{\sigma \xi}}=2 v_{0_{\sigma \tau}}-2 v_{0_{\xi \tau}}+a_{10}\left(-v_{0_{\sigma}}+v_{0_{\xi}}\right)+a_{01}\left(-w_{0_{\sigma}}+w_{0_{\xi}}\right) \\
+p_{1}\left(\sigma+\xi,-v_{0_{\sigma}}+v_{0_{\xi}},-w_{0_{\sigma}}+w_{0_{\xi}}\right)+a_{03}\left(-w_{0_{\sigma}}+w_{0_{\xi}}\right)^{3}, \\
-\infty<\sigma<\xi<\infty, \tau>0, \\
-4 w_{1_{\sigma \xi}}=2 w_{0_{\sigma \tau}}-2 w_{0_{\xi \tau}}+b_{01}\left(-w_{0_{\sigma}}+w_{0_{\xi}}\right)+p_{2}\left(\sigma+\xi,-v_{0_{\sigma}}+v_{0_{\xi}},-w_{0_{\sigma}}+w_{0_{\xi}}\right) \\
+b_{03}\left(-w_{0_{\sigma}}+w_{0_{\xi}}\right)^{3}, \quad-\infty<\sigma<\xi<\infty, \tau>0, \\
v_{1}(\sigma, \xi, \tau)=V_{01} \sin (m \sigma), \quad-\infty<\sigma=\xi<\infty, \tau=0, \\
w_{1}(\sigma, \xi, \tau)=W_{01} \sin (n \sigma), \quad-\infty<\sigma=\xi<\infty, \tau=0, \\
-v_{1_{\sigma}}(\sigma, \xi, \tau)+v_{1_{\xi}}(\sigma, \xi, \tau)=-v_{0_{\tau}}(\sigma, \xi, \tau)+V_{11} \sin (m \sigma), \quad-\infty<\sigma=\xi<\infty, \tau=0, \\
\left.-w_{1_{\sigma}}(\sigma, \xi, \tau)+w_{1_{\xi}}(\sigma, \xi, \tau)=-w_{0_{\tau}}(\sigma, \xi, \tau)+W_{11} \sin (n \sigma), \quad-\infty<\sigma=\xi<\infty, \tau=0.23\right)
\end{gathered}
$$

Furthermore, $v_{0}=w_{0}=v_{1}=w_{1}=0$ if $\sigma=k \pi-\theta, \xi=k \pi+\theta$, and $\tau=\varepsilon \theta$ with $k \in \mathbb{Z}$ and $\theta \geq 0$. In the further analysis $v_{0}, w_{0}, v_{1}$, and $w_{1}$ will be determined, and it will be shown that the formal approximation $\mathbf{u}_{A}(x, t ; \varepsilon) \equiv\left(v_{0}(x-t, x+t, \varepsilon t)+\varepsilon v_{1}(x-t, x+\right.$ $\left.t, \varepsilon t), w_{0}(x-t, x+t, \varepsilon t)+\varepsilon w_{1}(x-t, x+t, \varepsilon t)\right)^{\mathrm{T}}$ is an order $\varepsilon$ asymptotic approximation (as $\varepsilon \rightarrow 0)$ of the solution $\mathbf{u}(x, t)$ of the initial-boundary value problem $(5.1)-(5.7)$ for $0 \leq x \leq \pi$ and $0 \leq t \leq L|\varepsilon|^{-1}$. 
The general solutions of the partial differential equations (5.14) and (5.15) are given by $v_{0}(\sigma, \xi, \tau)=h_{0}(\sigma, \tau)+k_{0}(\xi, \tau)$ and $w_{0}(\sigma, \xi, \tau)=f_{0}(\sigma, \tau)+g_{0}(\xi, \tau)$, respectively. The initial values (5.16)-(5.19) imply that $h_{0}, k_{0}, f_{0}$, and $g_{0}$ have to satisfy $h_{0}(\sigma, 0)+k_{0}(\sigma, 0)=V_{00} \sin (m \sigma),-h_{0}^{\prime}(\sigma, 0)+k_{0}^{\prime}(\sigma, 0)=V_{10} \sin (m \sigma), f_{0}(\sigma, 0)+$ $g_{0}(\sigma, 0)=W_{00} \sin (n \sigma)$, and $-f_{0}^{\prime}(\sigma, 0)+g_{0}^{\prime}(\sigma, 0)=W_{10} \sin (n \sigma)$, where the prime denotes differentiation with respect to the first argument. From the odd and $2 \pi$-periodic extension in $x$ it follows indirectly that $h_{0}, k_{0}, f_{0}$, and $g_{0}$ have to satisfy $k_{0}(\sigma, \tau)=$ $-h_{0}(-\sigma, \tau), h_{0}(\sigma, \tau)=h_{0}(\sigma+2 \pi, \tau), g_{0}(\sigma, \tau)=-f_{0}(-\sigma, \tau)$, and $f_{0}(\sigma, \tau)=f_{0}(\sigma+2 \pi, \tau)$ for $-\infty<\sigma<\infty$ and $\tau \geq 0$. The undetermined behaviour of $h_{0}$ and $f_{0}$ with respect to $\tau$ will be used to avoid secular terms in $v_{1}$ and $w_{1}$. From the well-posedness theorem it follows that $\mathbf{u}, \mathbf{u}_{t}$, and $\mathbf{u}_{x}$ are $O(1)$ on $\Omega_{L}$. So, $\tilde{\mathbf{u}}$ and its first derivatives have to remain $O(1)$ on $-\infty<x<\infty$ and $0 \leq t \leq L|\varepsilon|^{-1}$. Furthermore, it should be noticed that the equations for $v_{0}, w_{0}, v_{1}$, and $w_{1}$ have been derived under the assumption that $v_{0}, w_{0}, v_{1}, w_{1}$ and their derivatives up to order two are $O(1)$. These boundedness conditions on $v_{0}, w_{0}, v_{1}$, and $w_{1}$ determine the behaviour of $h_{0}$ and $f_{0}$ with respect to $\tau$. From (5.20)-(5.25), $v_{1_{\sigma}}, v_{1_{\xi}}, w_{1_{\sigma}}$, and $w_{1_{\xi}}$ may be obtained easily. For instance,

$$
\begin{aligned}
& -4 w_{1_{\sigma}}(\sigma, \xi, \tau)=-4 w_{1_{\sigma}}(\sigma, \sigma, \tau)+(\xi-\sigma)\left(2 f_{0_{\sigma \tau}}(\sigma, \tau)-b_{01} f_{0_{\sigma}}(\sigma, \tau)-b_{03} f_{0_{\sigma}}^{3}(\sigma, \tau)\right) \\
& -3 b_{03} f_{0_{\sigma}}(\sigma, \tau) \int_{\sigma}^{\xi} g_{0_{\theta}}^{2}(\theta, \tau) d \theta+\int_{\sigma}^{\xi}\left\{-2 g_{0_{\theta \tau}}(\theta, \tau)+b_{01} g_{0_{\theta}}(\theta, \tau)\right. \\
& \left.+3 b_{03} f_{0_{\sigma}}^{2}(\sigma, \tau) g_{0_{\theta}}(\theta, \tau)+b_{03} g_{0_{\theta}}^{3}(\theta, \tau)\right\} d \theta \\
& +\int_{\sigma}^{\xi} p_{2}\left(\sigma+\theta,-h_{0_{\sigma}}(\sigma, \tau)+k_{0_{\theta}}(\theta, \tau),-f_{0_{\sigma}}(\sigma, \tau)+g_{0_{\theta}}(\theta, \tau)\right) d \theta+h^{*}(\sigma, \tau),
\end{aligned}
$$

where $h^{*}$ will be determined later. In (5.26) the integral with integrand $p_{2}$ is of $O(1)$ for all values of $\sigma$ and $\xi$, because the function $p_{2}$ of $O(1)$ is $4 \pi$-periodic in $\theta$ and the integral over such a period is equal to zero. Since the first integral in (5.26) contains a nonnegative and $2 \pi$-periodic integrand it follows that this integral will grow with the length $\xi-\sigma$ of the integration interval. It turns out that this integral can be written in a part which is of $O(1)$ for all values of $\sigma$ and $\xi$, and in a part which is linear in $\xi-\sigma$ :

$$
\int_{\sigma}^{\xi} g_{0_{\theta}}^{2}(\theta, \tau) d \theta=\int_{\sigma}^{\xi}\left\{g_{0_{\theta}}^{2}(\theta, \tau)-\frac{1}{2 \pi} \int_{0}^{2 \pi} g_{0_{\psi}}^{2}(\psi, \tau) d \psi\right\} d \theta+\frac{\xi-\sigma}{2 \pi} \int_{0}^{2 \pi} g_{0_{\psi}}^{2}(\psi, \tau) d \psi
$$

Noticing that $\xi-\sigma=2 t$ it follows that $\xi-\sigma$ is of $O\left(|\varepsilon|^{-1}\right)$ on a time scale of $O\left(|\varepsilon|^{-1}\right)$. So, $w_{1_{\sigma}}$ will be of $O\left(|\varepsilon|^{-1}\right)$ unless $f_{0}$ and $g_{0}$ are such that in (5.26) the terms of $O\left(|\varepsilon|^{-1}\right)$ (that is, terms linear in $\xi-\sigma$ ) disappear. It turns out that $w_{1_{\sigma}}, w_{1_{\xi}}, v_{1_{\sigma}}$, and $v_{1_{\xi}}$ are all $O(1)$ on a time scale of $O\left(|\varepsilon|^{-1}\right)$ if $f_{0}(\sigma, \tau), g_{0}(\xi, \tau), h_{0}(\sigma, \tau)$, and $k_{0}(\xi, \tau)$ satisfy 
the following four conditions:

$$
\begin{array}{r}
2 f_{0_{\sigma \tau}}-b_{01} f_{0_{\sigma}}-b_{03} f_{0_{\sigma}}^{3}-\frac{3 b_{03}}{2 \pi} f_{0_{\sigma}} \int_{0}^{2 \pi} g_{0_{\theta}}^{2}(\theta, \tau) d \theta=0, \\
2 g_{0_{\xi \tau}}-b_{01} g_{0_{\xi}}-b_{03} g_{0_{\xi}}-\frac{3 b_{03}}{2 \pi} g_{0_{\xi}} \int_{0}^{2 \pi} f_{0_{\theta}}^{2}(\theta, \tau) d \theta=0, \\
2 h_{0_{\sigma \tau}}-a_{10} h_{0_{\sigma}}-a_{01} f_{0_{\sigma}}-a_{03} f_{0_{\sigma}}^{3}-\frac{3 a_{03}}{2 \pi} f_{0_{\sigma}} \int_{0}^{2 \pi} g_{0_{\theta}}^{2}(\theta, \tau) d \theta=0, \\
2 k_{0_{\xi \tau}}-a_{10} k_{0_{\xi}}-a_{01} g_{0_{\xi}}-a_{03} g_{0_{\xi}}^{3}-\frac{3 a_{03}}{2 \pi} g_{0_{\xi}} \int_{0}^{2 \pi} f_{0_{\theta}}^{2}(\theta, \tau) d \theta=0 .
\end{array}
$$

From $g_{0}(\theta, \tau)=-f_{0}(-\theta, \tau)$ and from $k_{0}(\theta, \tau)=-h_{0}(-\theta, \tau)$ it follows that the first and second condition as well as the third and fourth condition are equivalent. So, $w_{1_{\sigma}}, w_{1_{\xi}}, v_{1_{\sigma}}$, and $v_{1_{\xi}}$ are all $O(1)$ on a time scale of $O\left(|\varepsilon|^{-1}\right)$ if $f_{0}$ and $h_{0}$ satisfy

$$
2 f_{0_{\sigma \tau}}-b_{01} f_{0_{\sigma}}-b_{03} f_{0_{\sigma}}^{3}-\frac{3 b_{03}}{2 \pi} f_{0_{\sigma}} \int_{0}^{2 \pi} f_{0_{\theta}}^{2}(\theta, \tau) d \theta=0
$$

and

$$
2 h_{0 \sigma \tau}-a_{10} h_{0_{\sigma}}-a_{01} f_{0_{\sigma}}-a_{03} f_{0_{\sigma}}^{3}-\frac{3 a_{03}}{2 \pi} f_{0_{\sigma}} \int_{0}^{2 \pi} f_{0_{\theta}}^{2}(\theta, \tau) d \theta=0
$$

In $[3,7]$ an equation similar to Eq. (5.27) has been solved. If the method introduced in [3] is applied to Eq. (5.27) one obtains $f_{0}(\sigma, \tau)$ after some calculations, and so $w_{0}(\sigma, \xi, \tau)=f_{0}(\sigma, \tau)-f_{0}(-\xi, \tau)$. It turns out that $f_{0}$ and $w_{0}$ are given by

$$
\begin{aligned}
f_{0}(\sigma, \tau)= & \frac{\lambda(\tau)}{n \phi^{1 / 2}(\tau)} \arcsin \left[\left[\frac{W_{n} \phi(t)}{1+W_{n} \phi(\tau)}\right]^{1 / 2} \sin (\alpha+n \sigma)\right]+k^{*}(\tau), \\
w_{0}(\sigma, \xi, \tau)= & \frac{\lambda(\tau)}{n \phi^{1 / 2}(\tau)}\left\{\arcsin \left[\left[\frac{W_{n} \phi(\tau)}{1+W_{n} \phi(\tau)}\right]^{1 / 2} \sin (\alpha+n \sigma)\right]\right. \\
& \left.-\arcsin \left[\left[\frac{W_{n} \phi(\tau)}{1+W_{n} \phi(\tau)}\right]^{1 / 2} \sin (\alpha-n \xi)\right]\right\}
\end{aligned}
$$

where $k^{*}(\tau)$ is an arbitrary function in $\tau$ with $k^{*}(0)=0, \sigma=x-t, \xi=x+t$, $\tau=\varepsilon t, W_{n}=n^{2} W_{00}^{2}+W_{10}^{2}, \alpha$ is given by $\cos \alpha=n W_{00} W_{n}^{-1 / 2}$ and $\sin \alpha=W_{10} W_{n}^{-1 / 2}$, where $\lambda(\tau)$ and $\phi(\tau)$ are implicitly given by $\lambda(\tau)=4 m^{-3}(\tau) \exp \left(b_{01} \tau / 2\right)$ and $\phi(\tau)=$ $\left(m(\tau) / W_{n}\right) \times(m(\tau)-2)$ with $m(\tau)$ determined by $m^{8}(\tau)-\frac{8}{7} m^{7}(\tau)=2^{6} W_{n} b_{03} b_{01}^{-1}(1-$ $\left.\exp \left(b_{01} \tau\right)\right)+3 \cdot 2^{8} / 7$. Now the (with respect to $h_{0}$ ) linear partial differential equation 
(5.28) can be solved and one obtains $h_{0}$ after some calculations, and so $v_{0}(\sigma, \xi, \tau)=$ $h_{0}(\sigma, \tau)-h_{0}(-\xi, \tau)$. It turns out that $v_{0}$ is given by

$$
\begin{aligned}
& v_{0}(\sigma, \xi, \tau) \\
& =\frac{1}{2} \exp \left(\frac{a_{10}}{2} \tau\right)\left\{V_{00} \sin (m \sigma)+\frac{1}{m} V_{10} \cos (m \sigma)+V_{00} \sin (m \xi)-\frac{1}{m} V_{10} \cos (m \xi)\right\} \\
& +\frac{a_{03}}{b_{03}}\left\{w_{0}(\sigma, \xi, \tau)-\frac{1}{2} \exp \left(\frac{a_{10}}{2} \tau\right)\left(W_{00} \sin (n \sigma)+\frac{1}{n} W_{10} \cos (n \sigma)+W_{00} \sin (n \xi)\right.\right. \\
& \left.+\frac{1}{n}\left(W_{10} \cos (n \xi)\right)\right\} \\
& \left.a_{01}-\frac{a_{03}}{b_{03}} b_{01}+\frac{a_{03}}{b_{03}} a_{10}\right) \int_{0}^{\tau} \exp \left(\frac{a_{10}}{2}\left(\tau-\tau^{\prime}\right)\right) w_{0}\left(\sigma, \xi, \tau^{\prime}\right) d \tau^{\prime}
\end{aligned}
$$

where $w_{0}$ is given by (5.30). Now the linear initial-value problems $(5.20)-(5.25)$ for $v_{1}$ and $w_{1}$ can be solved, yielding

$$
\begin{array}{r}
v_{1}(\sigma, \xi, \tau)=-\frac{1}{4} \int_{\xi}^{\sigma} \int_{\psi}^{\xi} p_{1}\left(\psi+\phi,-h_{0_{\psi}}(\psi, \tau)+k_{0_{\phi}}(\phi, \tau),-f_{0_{\psi}}(\psi, \tau)+g_{0_{\phi}}(\phi, \tau)\right) d \phi d \psi \\
+\frac{3}{4} a_{03}\left(f_{0}(\sigma, \tau)+g_{0}(\xi, \tau)\right) \int_{\sigma}^{\xi}\left(f_{0_{\theta}}^{2}(\theta, \tau)-\frac{1}{2 \pi} \int_{0}^{2 \pi} f_{0_{\psi}}^{2}(\psi, \tau) d \psi\right) d \theta-\frac{3}{4} a_{03} \\
\times \int_{\sigma}^{\xi}\left[\left(f_{0_{\theta}}^{2}(\theta, \tau)-\frac{1}{2 \pi} \int_{0}^{2 \pi} f_{0_{\psi}}^{2}(\psi, \tau) d \psi\right)\left(f_{0}(\theta, 0)+g_{0}(\theta, 0)\right)\right] d \theta+h_{1}(\sigma, \tau)+k_{1}(\xi, \tau),
\end{array}
$$

and

$$
\begin{array}{r}
w_{1}(\sigma, \xi, \tau)=-\frac{1}{4} \int_{\xi}^{\sigma} \int_{\psi}^{\xi} p_{2}\left(\psi+\phi,-h_{0_{\psi}}(\psi, \tau)+k_{0_{\phi}}(\phi, \tau),-f_{0_{\psi}}(\psi, \tau)+g_{0_{\phi}}(\phi, \tau)\right) d \phi d \psi \\
+\frac{3}{4} b_{03}\left(f_{0}(\sigma, \tau)+g_{0}(\xi, \tau)\right) \int_{\sigma}^{\xi}\left(f_{0_{\theta}}^{2}(\theta, \tau)-\frac{1}{2 \pi} \int_{0}^{2 \pi} f_{0_{\psi}}^{2}(\psi, \tau) d \psi\right) d \theta-\frac{3}{4} b_{03} \\
\times \int_{\sigma}^{\xi}\left[\left(f_{0_{\theta}}^{2}(\theta, \tau)-\frac{1}{2 \pi} \int_{0}^{2 \pi} f_{0_{\psi}}^{2}(\psi, \tau) d \psi\right)\left(f_{0}(\theta, 0)+g_{0}(\theta, 0)\right)\right] d \theta+f_{1}(\sigma, \tau)+g_{1}(\xi, \tau),
\end{array}
$$

where (for $\sigma=\xi$ and $\tau=0) h_{1}+k_{1}$ and $f_{1}+g_{1}$ are determined by the initial values (5.22)-(5.25). The undetermined behaviour of $f_{1}, g_{1}, h_{1}$, and $k_{1}$ with respect to $\tau$ can be used to avoid secular terms in $v_{2}$ and $w_{2}$. However, in this analysis, $v_{2}$ and $w_{2}$ will not be determined. For that reason it may be assumed that $f_{1}=f_{1}(\sigma), g_{1}=g_{1}(\xi)$, $h_{1}=h_{1}(\sigma)$, and $k_{1}=k_{1}(\xi)$, and then

$$
\begin{aligned}
f_{1}(\sigma)+g_{1}(\xi)=-\frac{1}{2} \int_{\sigma}^{\xi} w_{0_{\tau}}(\theta, \theta, 0) d \theta+\frac{1}{2} W_{01}(\sin (n \sigma) & +\sin (n \xi)) \\
& +\frac{1}{2 n} W_{11}(\cos (n \sigma)-\cos (n \xi))
\end{aligned}
$$


and

$$
\begin{aligned}
h_{1}(\sigma)+k_{1}(\xi)=-\frac{1}{2} \int_{\sigma}^{\xi} v_{0_{\tau}}(\theta, \theta, 0) d \theta+\frac{1}{2} V_{01}(\sin (m \sigma) & +\sin (m \xi)) \\
& +\frac{1}{2 m} V_{11}(\cos (m \sigma)-\cos (m \xi)) .
\end{aligned}
$$

It can be shown from (5.30)-(5.33) that $v_{0}, v_{1}, w_{0}, w_{1}$ and their derivatives up to order two are of $O(1)$ for $-\infty<x<\infty$ and $0 \leq t \leq L|\varepsilon|^{-1}$. So, the assumptions under which the equations for $v_{0}, v_{1}, w_{0}$, and $w_{1}$ have been derived, are justified. So far a vector-valued function $\mathbf{u}_{A}(x, t ; \varepsilon) \equiv\left(v_{0}(x-t, x+t, \varepsilon t)+\varepsilon v_{1}(x-t, x+t, \varepsilon t)\right.$, $\left.w_{0}(x-t, x+t, \varepsilon t)+\varepsilon w_{1}(x-t, x+t, \varepsilon t)\right)^{\mathrm{T}}$ has been constructed. It can easily be seen that $\mathbf{u}_{A}$ satisfies (5.3), (5.4), and (5.7) exactly, and (5.5) and (5.6) up to order $\varepsilon^{2}$ in the sense of Theorem 3.1. After lengthy calculations it can also be shown that $\mathbf{u}_{A}$ satisfies $(5.1)$ and $(5.2)$ up to $\varepsilon^{2} \mathbf{c}_{1}(x, t ; \varepsilon) \equiv \varepsilon^{2}\left(c_{11}(x, t ; \varepsilon), c_{12}(x, t ; \varepsilon)\right)^{\mathrm{T}}$, where $c_{1 i}, \partial c_{1 i} / \partial x \in C\left(\Omega_{L} \times\left[-\varepsilon_{0}, \varepsilon_{0}\right], \mathbb{R}\right)$ for $i=1,2$ with $\mathbf{c}_{1}(0, t ; \varepsilon)=\mathbf{c}_{1}(\pi, \tau ; \varepsilon)=\mathbf{0}$ for $0 \leq t \leq L|\varepsilon|^{-1}$. Furthermore, it can be shown that $\mathrm{c}_{1}(x, t ; \varepsilon)$ and its derivative with respect to $x$ are uniformly bounded in $t$ and $\varepsilon$. Then it follows from Theorem 3.1 that $\mathbf{u}_{A}(x, t ; \varepsilon)$ is an order $\varepsilon$ asymptotic approximation $($ as $\varepsilon \rightarrow 0)$ of the solution of the initial-boundary value problem (5.1)-(5.7) for $0 \leq x \leq \pi$ and $0 \leq t \leq L|\varepsilon|^{-1}$, that is, $\left\|\mathbf{u}-\mathbf{u}_{A}\right\|_{C_{M_{1}}^{2}}=O(\varepsilon)$. From this estimate the following estimate can be obtained with $\mathbf{u}_{0}(x, t ; \varepsilon) \equiv\left(v_{0}(x-t, x+t, \varepsilon t), w_{0}(x-t, x+t, \varepsilon t)\right)^{\mathrm{T}}$ :

$$
\left\|\mathbf{u}-\mathbf{u}_{0}\right\|_{C_{\bar{M}_{1}}^{2}}=\left\|\mathbf{u}-\mathbf{u}_{A}+\mathbf{u}_{A}-\mathbf{u}_{0}\right\|_{C_{\bar{M}_{1}}^{2}} \leq\left\|\mathbf{u}-\mathbf{u}_{A}\right\|_{C_{\bar{M}_{1}}^{2}}+\left\|\mathbf{u}_{A}-\mathbf{u}_{0}\right\|_{C_{M_{1}}^{2}}=O(\varepsilon) .
$$

Hence, $\left(v_{0}(x-t, x+t, \varepsilon t), w_{0}(x-t, x+t, \varepsilon t)\right)^{\mathrm{T}}$, where $v_{0}$ and $w_{0}$ are given by (5.31) and (5.30), respectively, is also an order $\varepsilon$ asymptotic approximation (as $\varepsilon \rightarrow 0)$ of the solution $\mathbf{u}(x, t ; \varepsilon)$ of problem (5.1)-(5.7) for $0 \leq x \leq \pi$ and $0 \leq t \leq L|\varepsilon|^{-1}$, in which $L$ is an $\varepsilon$-independent, positive constant.

6. Some general remarks. The asymptotic theory presented in Secs. 2 and 3 can readily be extended to other types of initial-boundary value problems. For instance, if the initial-boundary value problem $(2.1)-(2.4)$ is considered where the boundary conditions (2.4) are replaced by $\mathbf{u}(0, t)=\mathbf{0}$ and $\mathbf{u}_{x}(\pi, t)=\mathbf{0}$ (a fixed end condition at $x=0$ and a free end condition at $x=\pi$ ) then an integral equation equivalent with this problem is needed to prove the well-posedness of the problem and the asymptotic validity of a class of formal approximations. To obtain this equivalent integral equation the initial-boundary value problem should be extended to an initial value problem. This can be accomplished by extending the dependent variable $\mathbf{u}$, the nonlinearity and the initial values in $x$, such that these functions are odd about $x=0$, even about $x=\pi$ and $4 \pi$-periodic with respect to $x$. In this way the equivalent integral equation is obtained and the techniques applied in Secs. 2 and 3 can again be used to prove the well-posedness of the problem and the asymptotic validity of formal approximations on $\varepsilon$-dependent time scales.

In Sec. 4 the assumption is made that the cross section of the circular conductor with small ice ridge is symmetric. However, this assumption is not necessary. In fact, for an arbitrary profile, galloping may occur if the lift- and drag-coefficients 
$c_{L}(\alpha)$ and $c_{D}(\alpha)$ are such that there exists an interval in $\alpha$ with $\alpha_{0}<\alpha<\alpha_{2}$ for which the den Hartog criterion $c_{D}(\alpha)+d c_{L}(\alpha) / d \alpha<0$ is satisfied. Then, the static angle of attack $\alpha_{s}$ can be chosen such that galloping may set in. It should be noted that the analysis in Secs. 4 and 5 is correct if there exists an angle $\alpha_{1}$ with $c_{L}\left(\alpha_{1}\right)=0$ and $\alpha_{0}<\alpha_{1}<\alpha_{2}$.

In Sec. 5 the model is studied, which has been derived in Sec. 4. It is assumed in Sec. 5 that $\mathbf{u}(x, t)$ is a twice continuously differentiable function. This assumption can be justified as follows. The velocities $v_{t}$ and $w_{t}$ of the conductor in the horizontal and in the vertical direction are continuous in $x$ and $t$, and the aerodynamic coefficients $c_{D}(\alpha)$ and $c_{L}(\alpha)$ are continuous in $\alpha$. Since $\alpha=\alpha_{s}+\phi=\alpha_{s}+\arctan \left(-w_{t}\left(v_{\infty}-v_{t}\right)^{-1}\right)$ with $\left|w_{t}\right|<v_{\infty}$ and $\left|v_{t}\right|<v_{\infty}$ it follows that the right-hand sides of Eqs. (4.1) and (4.2) are continuous in $x$ and $t$. In the left-hand sides of Eqs. (4.1) and (4.2) the terms

$$
\frac{\partial}{\partial x}\left\{v_{x}\left(1+v_{x}^{2}+w_{x}^{2}\right)^{-1 / 2}\right\} \text { and } \frac{\partial}{\partial x}\left\{w_{x}\left(1+v_{x}^{2}+w_{x}^{2}\right)^{-1 / 2}\right\}
$$

represent in fact the curvature of the transmission line. Since it is natural to assume that the curvature is continuous in $x$ and $t$, it follows that $v_{t t}$ and $w_{t t}$ should be continuous in $x$ and $t$. So, it is more or less natural to assume that $\mathbf{u}(x, t)=$ $(v(x, t), w(x, t))^{\mathrm{T}}$ should be twice continuously differentiable with respect to $x$ and $t$.

In Sec. 5 monochromatic initial values have been considered, because the galloping oscillations often affect only a single mode of vibration [14]. To obtain some information about the oscillation amplitudes the following formulas can be used:

$$
\begin{gathered}
v(x, t)=\frac{-\rho_{a} d v_{\infty}^{2} a_{00}}{4 T A} x(x-l)+\frac{l v_{\infty}}{\pi c} \bar{v}\left(\frac{\pi}{l} x, \frac{\pi c}{l} t\right), \\
w(x, t)=\frac{-\rho_{a} d v_{\infty}^{2} b_{00}}{4 T A} x(x-l)+\frac{l v_{\infty}}{\pi c} \bar{w}\left(\frac{\pi}{l} x, \frac{\pi c}{l} t\right),
\end{gathered}
$$

where $v(x, t), w(x, t), \rho_{a}, d, v_{\infty}, c, T, A, l, \bar{v}, \bar{w}, a_{00}$, and $b_{00}$ are defined as in Sec. 4. The first terms in these formulas may be considered as the position of the conductor in rest, whereas the second terms represent the change of the position of the conductor due to galloping. For large values of $t$ (that is, $\varepsilon t=\tau \rightarrow \infty$ ) it can be shown from (5.30) that $w_{0}$ (the first-order approximation of $\bar{w}$ ) tends to a standing triangular wave with amplitude $(\pi /(2 n))\left(b_{01} /-b_{02}\right)^{1 / 2}$ and period $2 \pi / n$. It should be noted that for these values of $t$ little can be said about the asymptotic validity of the results, since only for finite values of $\varepsilon t$, that is, for $0 \leq \tau=\varepsilon t \leq L<\infty$, the asymptotic validity of the results could be established. It is also interesting to mention that it can be shown from (5.31) that

$$
\lim _{\tau \rightarrow \infty} v_{0}(\sigma, \xi, \tau)=\left(\frac{a_{03} b_{01}-a_{01} b_{03}}{a_{10} b_{03}}\right) \lim _{\tau \rightarrow \infty} w_{0}(\sigma, \xi, \tau) .
$$

These results imply that the maximum oscillation amplitudes due to galloping may be approximated by

$$
\frac{l v_{\infty}}{2 n c}\left(\frac{-c_{D 0}-c_{L 1}}{\frac{1}{2} c_{D 0}+\frac{1}{6} c_{L 1}+c_{D 2}+c_{L 3}}\right)^{1 / 2}
$$


in the vertical direction, and by

$$
\frac{l v_{\infty}}{2 n c}\left(\frac{-c_{D 0}-c_{L 1}}{\frac{1}{2} c_{D 0}+\frac{1}{6} c_{L 1}+c_{D 2}+c_{L 3}}\right)^{1 / 2}\left(\frac{-c_{D 1}\left(\frac{1}{3} c_{D 0}+c_{D 2}+c_{L 3}\right)+\left(c_{D 3}-c_{L 2}\right)\left(c_{D 0}+c_{L 1}\right)}{2 c_{D 0}\left(\frac{1}{2} c_{D 0}+\frac{1}{6} c_{L 1}+c_{D 2}+c_{L 3}\right)}\right)
$$

in the horizontal direction, where $c_{D 0}, c_{D 1}, \ldots, c_{L 3}$ are the aerodynamic coefficients, which may be obtained from wind-tunnel measurements. In a practical application the quantity $\left(a_{03} b_{01}-a_{01} b_{03}\right)\left(a_{10} b_{03}\right)^{-1}$ is small compared to one. This implies that the amplitude of the horizontal oscillation is small compared to the amplitude of the vertical oscillation. This phenomenon, that galloping is an almost purely vertical oscillation, has also been noticed in nature [15].

In Sec. 4 it has been assumed that the tension $T$ in the conductor is constant. In [8] it has been shown for the free vibrations of a suspended cable for which the sagto-span ratio is small that the assumption is valid if the cable oscillates in a so-called antisymmetric in-plane mode. For the monochromatic initial values considered in Sec. 5 this implies that $n$ should be even. If the cable oscillates in a symmetric in-plane mode (that is, $n$ is odd) the assumption that $T$ is constant is only valid for the higher modes of vibration. For the lower modes of vibration with $n$ odd, the validity of the assumption that $T$ is constant heavily depends on the elastic properties of the conductor and the sag-span ratios (see [8]). However, in [15] it has been remarked that the most troublesome galloping mode is the $S$-shaped vertical mode of the conductor catenary, that is, $n$ is equal to 2 . So, it may be concluded that for this $S$-shaped mode the model (describing the galloping oscillations of overhead transmission lines) can be justified. However, if $n=1$ the assumption that $T$ is constant, is incorrect or at least doubtful.

In Sec. 5 monochromatic initial values have been considered in the vertical and in the horizontal direction. It can be shown that the analysis given in Sec. 5 also can be applied if the initial values in the horizontal direction consist of an arbitrary number of modes, that is, if $\bar{v}_{0}(x ; \varepsilon)=\sum_{k=1}^{\infty} a_{k}(\varepsilon) \sin (k x)$ and $\bar{v}_{1}(x ; \varepsilon)=\sum_{k=1}^{\infty} b_{k}(\varepsilon) \sin (k x)$, where $a_{k}(\varepsilon)$ and $b_{k}(\varepsilon)$ are such that differentiation and summation, and integration and summation, may be interchanged as often as is required. It then turns out that $v_{0}(\sigma, \xi, \tau)$ is given by

$$
\begin{aligned}
v_{0}(\sigma, \xi, \tau)= & \frac{1}{2} \exp \left(\frac{a_{10}}{2} \tau\right)\left\{\sum _ { k = 1 } ^ { \infty } \left(a_{k}(0) \sin (k \sigma)+\frac{b_{k}(0)}{k} \cos (k \sigma)\right.\right. \\
& \left.+a_{k}(0) \sin (k \xi)-\frac{b_{k}(0)}{k} \cos (k \xi)\right\}+\frac{a_{03}}{b_{03}}\left\{w_{0}(\sigma, \xi, \tau)-\frac{1}{2} \exp \left(\frac{a_{10}}{2} \tau\right)\right. \\
& \times\left(W_{00} \sin (n \sigma)+\frac{W_{10}}{n} \cos (n \sigma)+W_{00} \sin (n \xi)-\frac{W_{10}}{n} \cos (n \xi)\right\} \\
+ & \frac{1}{2}\left(a_{01}-\frac{a_{03}}{b_{03}} b_{01}+\frac{a_{03}}{b_{03}} a_{10}\right) \int_{0}^{\tau} \exp \left(\frac{a_{10}}{2}\left(\tau-\tau^{\prime}\right)\right) w_{0}\left(\sigma, \xi, \tau^{\prime}\right) d \tau^{\prime},
\end{aligned}
$$

where $w_{0}(\sigma, \xi, \tau)$ is given by (5.30). Since $a_{10}<0$ it follows from this formula that (for $\tau \rightarrow \infty$ ) the modes initially present in the horizontal direction disappear, and that $v_{0}$ tends to a standing triangular wave (see $(6.1)$ ), which is in fact determined by 
the vibration mode initially present in the vertical direction. After having obtained the approximation for the oscillation amplitudes (and the velocities of the conductor) it should always be checked if the values of $\alpha=\alpha_{1}+\arctan \left(-w_{t}\left(v_{\infty}-v_{t}\right)^{-1}\right)$ are such that the approximations (4.5) and (4.6) for $c_{D}(\alpha)$ and $c_{L}(\alpha)$ are still valid. It turns out that for the $c_{D}$ - and $c_{L}$-curve given in Fig. 4.2 and for the typical values of the physical quantities $l, d, v_{\infty}, c$, etc. (given in Sec. 4) the values of $\alpha$ are such that the approximations (4.5) and (4.6) for $c_{D}(\alpha)$ and $c_{L}(\alpha)$ are valid, and so these approximations are justified.

Acknowledgment. The author wishes to thank Prof. J. W. Reyn, Dr. A. H. P. van der Burgh, and Dr. J. G. Besjes for their careful reading of the manuscript. The author is especially grateful to Dr. Van der Burgh for his stimulating interest in the investigations. This research project was supported by the Netherlands Foundation for Mathematics SMC with financial aid from the Netherlands Organization for the Advancement of Pure Research (ZWO).

\section{REFERENCES}

[1] C. G. A. van der Beek and A. H. P. van der Burgh, On the periodic wind-induced vibrations of an oscillator with two degrees of freedom, Nieuw Arch. Wisk. 2, 207-225 (1987)

[2] J. G. Besjes, On the asymptotic methods for non-linear differential equations, J. Mécanique 8, 357-372 (1969)

[3] S. C. Chikwendu and J. Kevorkian, A perturbation method for hyperbolic equations with small nonlinearities, SIAM J. Appl. Math. 22, 235-258 (1972)

[4] W. Eckhaus, New approach to the asymptotic theory of nonlinear oscillations and wave-propagation, J. Math. Anal. Appl. 49, 575-611 (1975)

[5] J. P. den Hartog, Mechanical vibrations, 4th ed., McGraw-Hill, New York, 1956

[6] W. T. van Horssen and A. H. P. van der Burgh, On initial-boundary value problems for weakly semilinear telegraph equations. Asymptotic theory and application, SIAM J. Appl. Math. 48, 719-736 (1988)

[7] W. T. van Horssen, An asymptotic theory for a class of initial-boundary value problems for weakly nonlinear wave equations with an application to a model of the galloping oscillations of overhead transmission lines, SIAM J. Appl. Math. 48, 1227-1243 (1988)

[8] H. M. Irvine and T. K. Cauchey, The linear theory of free vibrations of a suspended cable, Proc. Roy. Soc. London Ser. A 341, 299-315 (1974)

[9] J. B. Keller and S. Kogelman, Asymptotic solutions of initial value problems for nonlinear partial differential equations, SIAM J. Appl. Math. 18, 748-758 (1970)

[10] J. Kevorkian and J. D. Cole, Perturbation methods in applied mathematics, Springer-Verlag, New York, 1981

[11] C. J. Myerscough, A simple model of the growth of wind-induced oscillations in overhead lines, J. Sound Vibration 28, 699-713 (1973)

[12] C. J. Myerscough, Further studies of the growth of wind-induced oscillations in overhead lines, J. Sound Vibration 39, 503-517 (1975)

[13] H. H. Ottens and R. K. Hack, Results of an exploratory study of the galloping oscillations of overhead transmission lines (in Dutch), Report NLR TR 80016 L of the National Aerospace Laboratory NLR, The Netherlands (1980)

[14] J. A. Sanders and F. Verhulst, Averaging methods in nonlinear dynamical systems, Appl. Math. Sci. 59, Springer-Verlag, New York, 1985

[15] A. Simpson, Wind-induced vibration of overhead power transmission lines, Sci. Prog. Oxford 68, 285-308 (1983)

[16] O. Vejvoda, Partial differential equations: time-periodic solutions, Martinus Nijhoff Publishers, The Hague, 1982 